\title{
An Efficient Wireless Extension Point Placement Algorithm in Urban Rectilineal WLANs
}

\author{
Aaron So, Student Member, IEEE and Ben Liang, Senior Member, IEEE
}

\begin{abstract}
With a small amount of software modification, wireless Extension Points (EPs), which are now commercially available, can be used to improve the throughput capacity of a Wireless Local Area Network (WLAN). An EP is an immobile device that has access to the power supply or is equipped with a high capacity battery but does not have direct access to the Internet. They relay data wirelessly between the Access Point (AP) and the mobile hosts. In this paper, we investigate the optimal placement of EPs such that the throughput capacity of an urban rectilineal WLAN can be maximized. Two channel models are studied, based on the Shannon capacity bound and IEEE 802.11 specifications. We first formulate a solution for the optimal EP placement problem in a general network scenario as a non-linear programming problem, then we propose an efficient algorithm that determines the optimal locations of a fixed number of EPs in a rectilineal network. Our results show that, for a wide range of system parameters, the optimally placed EPs can significantly increase the network throughput capacity. Moreover, we study how the number of EPs, transmission power, path loss exponent, channel models and traffic characteristics affect the optimal EP placement and expected throughput capacity of the network.
\end{abstract}

Index Terms-wireless local area networks, access point, extension point, optimal placement, capacity improvement, mathematical programming/optimization.

\section{INTRODUCTION}

In the near future, wireless access to the Internet is expected to become a necessity. Since wireless networks are expected to create a plethora of business opportunities, Wireless Local Area Network (WLAN) standards such as IEEE 802.11 and HiperLAN/2 which provide wireless broadband data access for mobile Internet users were developed. Moreover, products that support these standards and public hotspots have proliferated. Currently, hotspot service providers only provide WLAN broadband data services in locations such as coffee shops, hotels, and other small confined areas. Because of WLAN's frugality and high bandwidth, hotspot service providers are expected to expand their service coverage area to the urban environments in the near future. By then, WLAN users can not only enjoy high bandwidth Internet access in most indoor environments, but also on the streets and even inside vehicles.

Currently, the most commonly implemented WLANs in North America are based on the IEEE 802.11b standard. It uses Direct Sequence Spread Spectrum (DSSS) and is capable of

Aaron So and Ben Liang are with the Department of Electrical and Computer Engineering, University of Toronto, 10 King's College Road, Toronto, Ontario, M5S 3G4, Canada. E-mail: \{aaronso,liang\}@comm.utoronto.ca. This work has been supported in part by a grant from the Natural Sciences and Engineering Research Council of Canada and Bell Canada through its Bell University Laboratories R\&D program. supporting bit rates up to $11 \mathrm{Mbps}$. Two more recent standards, IEEE $802.11 \mathrm{a}$ and $802.11 \mathrm{~g}$, which are based on Orthogonal Frequency Division Multiplexing (OFDM), are capable of supporting up to 54Mbps data rates. The increase in bit rate is heavily motivated by the demand for content-rich web-based applications. For both real-time and non-real time applications, the user data rate requirements have increased dramatically in recent years. To satisfy the increasing demand of mobile users, new strategies are needed to improve the performance of the WLAN technologies. The main objective of this work is to increase the average bit rate between the access point and the mobile host.

A prodigious amount of effort has been spent on improving the capacity of wireless networks within the physical layer. Techniques such as array antennas, space time coding [1], and adaptive beam forming [2] are promising techniques that are often described as the final frontier of wireless communications. However, by using these techniques, the achievable bit rate is still limited by the received signal strength. Since the transmitter power is heavily regulated, little can be done within these techniques to improve the receive signal strength.

To alleviate this problem, one obvious technique to improve network capacity is to use an intermediate node to boost the power of a wireless signal, thus forming a heterogeneous multihop wireless network. With slight software modification, commercially available wireless Extension Points (EPs), e.g. D-Link DWL-800AP+ and Linksys WRE54G, which were originally designed as relay nodes to extend the coverage area of an Access Point (AP), can now be used to improve WLAN capacity. EPs are usually immobile, have access to power supply (or are equipped with a high-capacity battery), but do not have direct access to the Internet. An EP helps deliver a packet from the AP to a distanced Mobile Host (MH) by first receiving the packet, and then retransmitting it to the $\mathrm{MH}$. Clearly, since the bit rate between a transmitter and a receiver is a fast decreasing function of the distance between them, by using an EP, the achieved bit rate can be much higher than the original one hop bit rate.

In this work, we study how to optimally use EPs to relay traffic in urban rectilineal WLANs that are expected to be installed on city streets. An example of such a WLAN architecture is shown in Fig. 1, where an AP is wired to a router in the ISP Distribution Network, and EPs are installed along the street. Mobile hosts can either communicate directly with the AP or indirectly to the AP via an EP.

Given immobile relaying nodes similar to EPs, innovative techniques have been proposed in the past, to balance the traffic load [3], to extend the coverage [4], and to enhance 


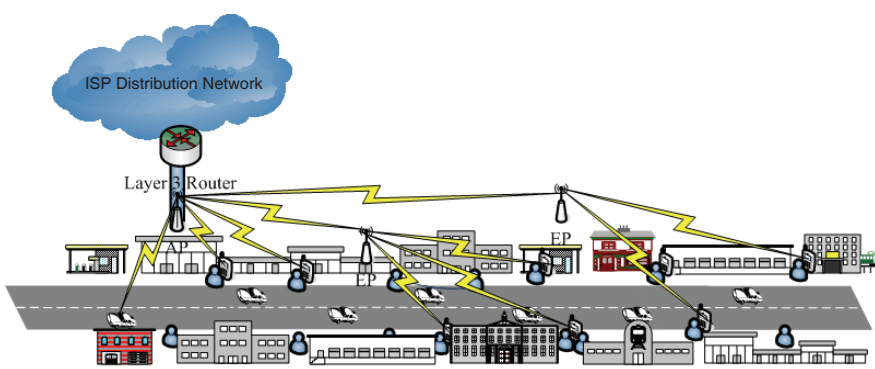

Fig. 1. A typical rectilineal WLAN in an urban environment.

the network capacity [5] of wireless networks. However, the potential function of EPs to improve capacity has not received sufficient attention. In particular, the question of how to place the EPs such that the throughput capacity of the network is optimized has not been answered.

In this work, we study the throughput capacity improvement of using EPs under two channel models, based on the Shannon capacity bound and IEEE $802.11 \mathrm{~g}$ data rates under fading. We propose an efficient algorithm to compute the optimal placement of EPs, and study how the number of EPs, transmission power, path loss exponent, channel models and traffic characteristics affect the optimal EP placement and expected throughput capacity of the network. By using the strategy we propose, hotspot service providers can accommodate more users into their networks without sacrificing the quality-ofservice that they provide. This, in turn, decreases the cost of wireless data transaction and accelerates the deployment of large-scale WLAN infrastructure.

The rest of this paper is organized as follows. In Section II, we review the related work in multihop wireless networks. In Section III, we describe the system model, define the network throughput capacity, derive the design objective of our placement algorithm, and explain the channel models we used in this work. In Section IV, we present the optimization model, a general solution formulation, and the proposed optimal EP placement algorithm. In Section V, we discuss the effect of different system parameters on the optimal placement of the EPs. Finally, in Section VI, we conclude.

\section{RELATED WORK}

Inspired by recent advances in ad hoc networking [6], the concept of using peer mobile hosts to relay data has been explored in the context of cellular networks. In [7], the concept of multihop cellular network was introduced. The results of this work shows that using peer mobile hosts to relay data to the base station can improve network performance when compared with the traditional single hop cellular network. Based on the approach of using mobile hosts to relay traffic, a routing protocols which emphasize on capacity maximization and energy efficiency have been proposed in [8] and [9] respectively. In more recent work, the problem of joint routing, link scheduling and power control in multihop wireless networks was investigated in [10], and the problem of joint rate allocation and transmission structure optimization for correlated data gathering in sensor networks was considered in [11].
Moreover, issues about frequency assignment and frequency recycling in such multihop networks have been addressed in [12], and the problem of joint routing and scheduling in a multi-radio, multi-channel, multihop wireless network has been studied [13].

The concept of using immobile nodes, which are refereed to as EPs in this paper, to relay traffic has received relatively less attention. EPs have several advantages when compared with mobile relay nodes. First, since EPs are relatively immobile ${ }^{1}$, it is reasonable to assume that they have access to external power supply or have a large built-in battery. Consequently, unlike in other works that use mobile hosts as relay nodes, energy is not a constraint for EPs. Second, since the distance between an AP and an EP is relatively static, links between the AP and the EPs should be relatively stable. Furthermore, the sedentary EPs can be configured to their optimal setting which maximizes their benefits.

The issues of using immobile relay node to relay traffic have been discussed in several other contexts. In [3], the $i C A R$ architecture for cellular network was introduced. $i C A R$ uses relay nodes to relay traffic from a congested cell to the less congested neighbor cells. The authors showed that the relays balance the traffic among cells and in turn improve the system performance. In [14] and [15], the authors proposed to use EPs in HiperLAN/2 and IEEE 802.11 systems, respectively. Their objective is to increase the coverage area provided by a single AP. The authors of [14] proposed a new MAC frame/subframe structure for a HiperLAN/2 system that takes the relay nodes into account. In [16], the authors evaluated this new MAC frame/sub-frame structure in a HiperLAN/2 system with EPs. They showed that the modified system not only extends the network coverage but also increases the capacity of the network significantly especially in environments where high attenuation is expected. In [17], the delay aspect of multihop network was investigated, and a contention free period leasing scheme based on the 802.11 PCF mode in a WLAN system with fixed relay nodes was proposed. The authors found that it is possible to support commercial standard voice services with a bounded delay if the number of hops from the AP is small.

From information theory research, the relay channel capacity was studied more than three decades ago in [18], and later considered by [19]. However, this capacity, which was intended for wired network, was derived based on the assumption that all nodes can transmit and receive simultaneously. Since such assumption is not realistic for the wireless medium, the wireless relay channel capacity, which assumes a node cannot transmit and receive at the same time, was recently derived in [20] and [21].

In [22], the expected throughput capacity of a wireless network with a single-tier relay infrastructure and the optimal placement of the relay nodes were derived based on the recent information theory results. We have shown in [22] that, under the additive white Gaussian noise channel model and with continuous bit rates, the optimally configured relay infrastructure can improve the throughput of a wireless network significantly.

\footnotetext{
${ }^{1}$ They can be moved to follow large time-scale network traffic variations.
} 


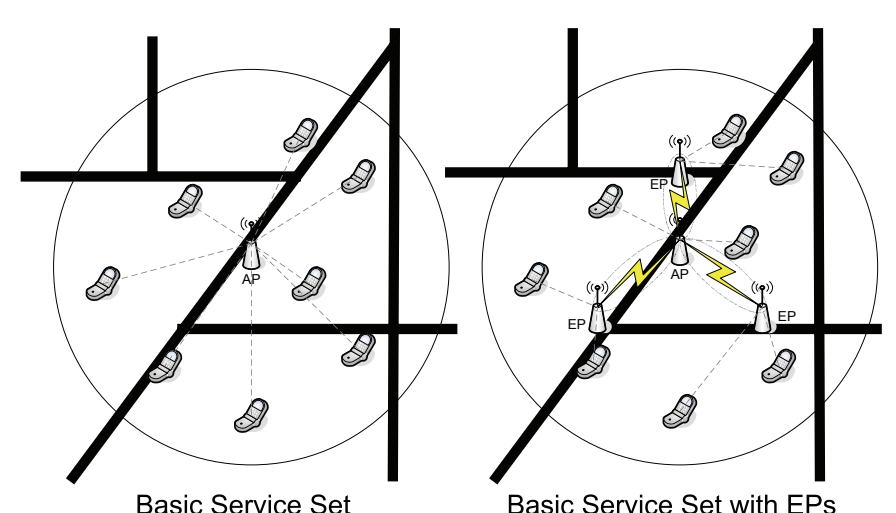

Fig. 2. Original BSS and BSS with EPs.

However, because of the finite modulation and coding schemes of modern communications systems, transmitters can only transmit in a finite set of bit rates. Moreover, in a wireless channel, multi-path fading has prominent effect on reliable communication between a transmitter receiver pair. To investigate the effect of relay infrastructure on network capacity in a practical wireless communications system is the main focus of this work. In particular, we explore the option of using commercially available extension points to perform relay with the objective of minimizing data packet transmission time. In addition, this paper considers a multi-tier EP architecture.

\section{EP Relaying ARChitecture AND Design OBJECTIVES}

We aim to improve the throughput capacity of a Basic Service Set (BSS) [23] by using wireless EPs. In a BSS, there is an AP connected with the wired ISP network, and this AP provides wireless coverage to a local area. Moreover, all MHs and the AP are fully connected (i.e., forming a complete graph) via a single channel. Thus, only one transmitter is allowed to transmit at any given time, and simultaneous transmission inside a BSS will result in collision.

In this work, we investigate relaying with wireless EPs in an environment where wiring is practically or economically infeasible to improve the throughput capacity of a BSS without using additional wireless resources. Therefore, the AP, EPs and MHs have to share the same wireless medium originally assigned to the BSS. Fig. 2 shows the difference between the original BSS and a BSS with EPs.

Since much research has been carried out on implementing relaying mechanisms [24] [25], in the next subsection, we provide only a high level description on how the system should operate. We then derive the objective function that will be optimized regarding the placement of the EPs. We would like to emphasize that our analytical and optimization framework has general applicability and is valid as long as the relaying mechanism follows the operation principles described in this section.

\section{A. Operational Procedure Overview}

To utilize the EPs intelligently, MHs locate at different locations should select the most appropriate EP to forward packets. ${ }^{2}$ If an EP is selected to assist a $\mathrm{MH}$, downlink packets from the wired network will be delivered from the AP to this $\mathrm{EP}$, and the EP will deliver the packet to the MH. The reverse is performed for uplink packets. In order to take full advantage of the EPs, a central entity, in this case, the AP, should have up-to-date link statistics and be able to make EP selection decisions for the MHs. Such information can be compiled in a report and send to the AP periodically. By collecting the link quality information, the AP can decide which EP (or the AP itself) each MH should select to maximize its throughput. The AP could inform the MH about its selection decision via a special control message or could embed such information in existing control packets such as RTS, CTS, ACK or POLL. We emphasize here that the exact implementation details can be flexible because the main focus of this work is on the optimal placement of EPs. In our analytical framework, we consider the case where a link quality estimation procedure exists and the AP and MHs are able to decide which EP to use in order to maximize their throughput. ${ }^{3}$

Once an EP is assigned to each $\mathrm{MH}$, a rate adaptation mechanism such as [26][27] has to be used by the $\mathrm{MH}$, $\mathrm{EP}$ and AP to decide the communication rate of each link. Again, the exact details of the rate adaptation scheme can be flexible. Our analytical framework only assumes that a rate adaptation mechanism is used, and the average packet transmission time between any pair of nodes is an increasing convex function of the distance between this two nodes. We do not expect the operation mechanism of the network to have high influence on the EP placement decision because in a fully connected network, packet collisions and other inefficiencies due to polling or distributed contention are mainly affected by the adopted protocols and the number of nodes in the network, but not the location of these nodes. ${ }^{4}$ In the next subsection, we will derive the objective of our optimization problem.

\section{B. Optimization Objective}

The sole benefit of using an EP to relay packets is to curtail the average data packet transmission time between the AP and the MHs. Therefore, to use the EPs optimally, they should be placed in locations resulting in the smallest average raw packet transmission time for different MHs. Before we express this optimization objective mathematically, let us consider the following system.

As shown in Fig. 3, for the simplest scenario, there is an access point located at the origin, a single MH located at $(x, y)$, and an EP located at $d$ meters from the beginning of a street. ${ }^{5}$ We denote the lengths of the uplink and downlink

\footnotetext{
${ }^{2}$ If none of the EPs are useful for a particular MH, this MH will not use any EP.

${ }^{3}$ The above information collection and EP selection procedure can be implemented in the Network Interface Card (NIC) software as an option for operation in a BSS with EPs. If a MH does not have this functionality, it will associate itself with the AP and behave as a MH in a regular BSS. Thus, the system is backward compatible.

${ }^{4}$ Since the network under investigation is fully connected, if any two nodes transmit simultaneously and result in a collision, the collision will occur no matter where these two nodes are placed.

${ }^{5}$ The subscript of the function $f$ and $g$ represents the street name, in this
} case street 1 . 


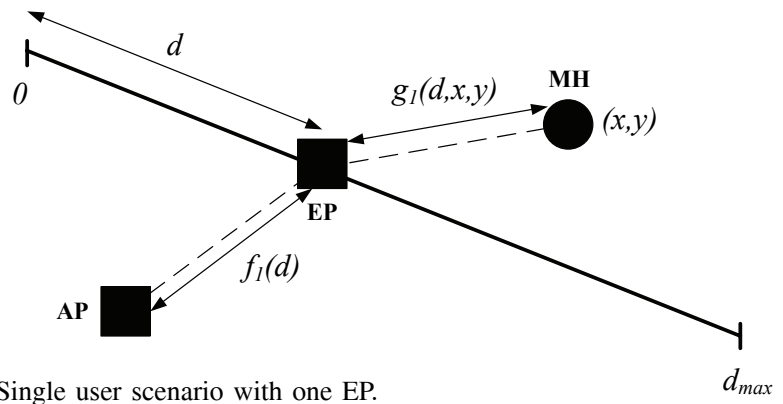

Fig. 3. Single user scenario with one EP.

packets by $z_{u}$ and $z_{d}$ respectively. The sizes of the packets correspond to the proportion of uplink and downlink traffic. The time axis is divided into time varying packet transaction cycles. At each cycle, the AP transmits a downlink packet to the $\mathrm{MH}$ via the $\mathrm{EP}$, and then the $\mathrm{MH}$ transmits an uplink packet to the AP also via the EP. Let $t(l, P, z)$ be a random variable representing the transmission time of an $z$-bit packet from a source with reference power $P$ to a destination $l$ meters away from the transmitter. Let us define the reference power of the AP, EP and MH be $P_{a}, P_{e}$ and $P_{m}$ respectively. Let $t_{e p}^{i}(x, y, d)$ be a random variable representing the time for a MH located at $(x, y)$ to complete the $i^{\text {th }}$ packet transaction cycle via the EP at $d$ meters from the beginning of street 1 . Then, we have

$$
\begin{aligned}
t_{e p}^{i}(x, y, d)= & t\left(f_{1}(d), P_{a}, z_{d}\right)+t\left(g_{1}(d, x, y), P_{e}, z_{d}\right)+ \\
& t\left(g_{1}(d, x, y), P_{m}, z_{u}\right)+t\left(f_{1}(d), P_{e}, z_{u}\right) .
\end{aligned}
$$

By the Law of Large Numbers [28], the throughput capacity of this MH with this particular EP placement is

$$
C(d)=\lim _{n \rightarrow \infty} \frac{n z}{\sum_{i=1}^{n} t_{e p}^{i}(x, y, d)}=\frac{z}{E\left[t_{e p}^{i}(x, y, d)\right]},
$$

where $z=z_{d}+z_{u}$. Therefore, in order to maximize the throughput capacity of the network, we need to find the optimal $d$ such that $E\left[t_{e p}^{i}(x, y, d)\right]$, which we call the expected packet transaction time in this paper, is minimized. Hence, our optimization objective is to find the optimal $d$ to minimize

$$
\begin{aligned}
T_{e p}(x, y, d)= & E\left[t_{e p}^{i}(x, y, d)\right] \\
= & E\left[t\left(f_{1}(d), P_{a}, z_{d}\right)+t\left(g_{1}(d, x, y), P_{e}, z_{d}\right)+\right. \\
& \left.t\left(g_{1}(d, x, y), P_{m}, z_{u}\right)+t\left(f_{1}(d), P_{e}, z_{u}\right)\right] \\
= & T\left(f_{1}(d), P_{a}, z_{d}\right)+T\left(g_{1}(d, x, y), P_{e}, z_{d}\right)+ \\
& T\left(g_{1}(d, x, y), P_{m}, z_{u}\right)+T\left(f_{1}(d), P_{e}, z_{u}\right),
\end{aligned}
$$

where $T(l, P, z)$ is the expected value of $t(l, P, z)$, which we called the expected packet transmission time.

In a network with multiple EPs, MHs will choose the most appropriate EP to use or communicate directly with the AP. Hence, MHs at different locations have different expected packet transaction times. Since in a typical WLAN environment, the locations of the MHs can be described only probabilistically, for a WLAN with multiple MHs, our minimization objective is the average expected packet transaction time in the network, which we called network expected packet transaction time.
In Section IV, we propose an optimization framework for the placement of EPs in a multi-user WLAN environment for a generic increasing convex function $T(l, P, z)$, which can be obtained from theoretical model or regression from site survey. But first, in the next subsection, we propose two theoretical models for the expected packet transmission time based on Shannon capacity and IEEE $802.11 \mathrm{~g}$ physical bit rates in a Rayleigh fading environment.

\section{Models for the Packet Transmission Time}

The packet transaction time is inversely proportional to the communication rate between a pair of transmitter and receiver. Since different random network parameters such as the distance between the transmitter and receiver and channel fading affect the reliable communication rate, the packet transaction time is random. In this paper, two transmission rate models are used: 1) the Shannon Capacity bound for a point-to-point Gaussian channel, and 2) the IEEE 802.11g physical layer model under Rayleigh fading. For both models, the following propagation path loss model is used [29]:

$$
P_{2}=\frac{P_{1}}{d_{2}^{\alpha}},
$$

where $P_{1}$ is the reference signal power measured at one meter away from the transmitter, $P_{2}$ is the signal power measured at $d_{2}$ meters away from the transmitter, and $\alpha$ is a positive constant representing the path loss roll off factor. The reference power $P_{1}$ can be obtained via field measurement or calculated using the following free space path loss formula [29].

$$
P_{1}=\frac{P_{t} G_{t} G_{r} \lambda^{2}}{(4 \pi)^{2} L}
$$

where $P_{t}$ is the transmitted power, $G_{t}$ is the transmitter antenna gain, $G_{r}$ is the receiver antenna gain, $\lambda$ is the wavelength of the transmitted signal, and $L(L \geq 1)$ is the loss factor not related to propagation. In this work, $L$ is set to 1 .

In the following, we describe how the two transmission rate models are used to determine the transmission time of a packet.

1) Shannon Capacity Bound: The Shannon capacity model is use to approximate the bit rate between a transmitter and a receiver in future wireless communication systems. In this ideal model, the bit rate between a pair of transmitter and receiver is represented by the Shannon capacity for a pointto-point Gaussian channel. We assume that the noise variance, $N_{t}$, is the same anywhere in the network. Let the signal power received by a receiver be $P_{r}$ and the available bandwidth be $W$. The channel capacity is given by [30]

$$
W \log _{2}\left(1+\frac{P_{r}}{N_{t}}\right) \text { bits per second . }
$$

If the transmitter reference power is $P$, while the distance between the transmitter and receiver is $l$, by using (4) and (6), the bit rate between this transmitter and receiver is

$$
W \log _{2}\left(1+\frac{P}{l^{\alpha} N_{t}}\right) \text { bits per second . }
$$


If the transmitter has $x$ bits to transmit to the receiver, the data transaction time is

$$
T_{s}(l, P, z)=\frac{z}{W \log _{2}\left(1+\frac{P}{l^{\alpha} N_{t}}\right)} \text { seconds }
$$

2) IEEE 802.11g under Rayleigh Fading: A more realistic transmission rate model is based on the IEEE $802.11 \mathrm{~g}$ bit rate model under Rayleigh fading. Suppose there are $M$ data rates, denoted as $r_{1}, r_{2}, \ldots, r_{M}$, supported by the physical layer. Reliable communication by using rate $r_{m}$ can be realized only if the signal strength at the receiver is above a certain threshold, say $\eta_{m}$. Consequently, for the set of $M$ data rates, there is a set of $M$ thresholds, $\eta_{1}, \ldots, \eta_{M}$, and we further define $\eta_{0}=0$ and $\eta_{M+1}=\infty$. For IEEE 802.11g, there are 11 different physical communication rates, and the minimal threshold for each bit rate is specified by the standard.

Under Rayleigh fading, the instantaneous power, $\gamma$, is exponentially distributed with the probability density function

$$
p(\gamma)=\frac{1}{P_{r}} e^{\frac{-\gamma}{P_{r}}}
$$

where $P_{r}$ is the average power of $\gamma$. Consequently, the probability that a transmitter with reference power $P$ can transmit at rate $r_{m}$, to a receiver at distance $l$, where $l>1$, is

$$
p\left(r_{m}, l, P\right)=\int_{\eta_{m}}^{\eta_{m+1}} \frac{l^{\alpha}}{P} e^{\frac{-\gamma l^{\alpha}}{P}} d \gamma
$$

where $m=1,2, \ldots, M$. Furthermore, in some instances, the receiver can be located in a deep faded area, i.e., is experiencing bad channel condition. The probability of these instances, where the transmitter cannot transmit in any data rate, is

$$
p_{f}(l, P)=\int_{\eta_{0}}^{\eta_{1}} \frac{l^{\alpha}}{P} e^{\frac{-\gamma l^{\alpha}}{P}} d \gamma
$$

while the probability that the transmitter can transmit successfully is

$$
p_{s}(l, P)=1-p_{f}(l, P)=\sum_{m=1}^{M} p\left(r_{m}, l, P\right)
$$

Unlike the ideal Shannon bound model, in the discreterate model with fading, the transmitter needs a small channel probing time, denoted $T_{\text {prob }}$, to test the channel and decide the transmission rate before the actual data transmission can take place. If the transmitter determines that the channel condition does not allow it to transmit at any rate, it will give up its transmit opportunity, and probe the channel again later. The wasted channel probing time add to the total packet transmission time.

Let $T(l, P, z)$ be a random variable that represents the transaction time of an $z$ bit packet, and let $S$ and $F$ be the events of "good" and "bad" channel states respectively. By the Law of Total Expectation [28], the expected value of this packet transaction time can be derived from

$$
\begin{aligned}
E[T(l, P, z)]= & E[T(l, P, z) \mid F] p_{f}(l, P)+ \\
& E[T(l, P, z) \mid S] p_{s}(l, P) \\
= & {\left[E[T(l, P, z)]+T_{\text {prob }}\right] p_{f}(l, P)+} \\
& {\left[\sum_{m=1}^{M} \frac{p\left(r_{m}, l, P\right)}{p_{s}(l, P)}\left[\frac{z}{r_{m}}+T_{\text {prob }}\right]\right] \times p_{s}(l, P) . }
\end{aligned}
$$

In the above formulation, $E[T(l, P, z) \mid F]$ represents the expected packet transmission time given the current transmission fails. In this case, the probing time is wasted. Therefore, the conditional expected packet transmission time is the expected packet transmission time plus the wasted probing time, i.e., $\left[E[T(l, P, z)]+T_{\text {prob }}\right]$. Similarly, $E[T(l, P, z) \mid S]$ represents the expected packet transmission time given the current transmission is successful. Even though the transmission is successful, the packet can be transmitted at different rate according to the channel condition. In this case, the term $\frac{p\left(r_{m}, l, P\right)}{p_{s}(l, P)}$ represents the probability that the packet can be transmitted at rate $r_{m}$, and the corresponding transmission time is $\left[\frac{z}{r_{m}}+\right.$ $\left.T_{\text {prob }}\right]$. Finally, since the expected packet transmission time is defined recursively, by rearranging the above, we have the data transaction time

$T_{g}(l, P, z)=E[T(l, P, z)]=\frac{T_{p r o b}}{p_{s}(l, P)}+\sum_{m=1}^{M} \frac{p\left(r_{m}, l, P\right)}{p_{s}(l, P)} \frac{z}{r_{m}}$.

\section{Optimal Placement of EPS}

In this section, we study how the placement of EPs affects the network expected packet transaction time. We first provide a generic optimization framework for the EP placement problem. Then, we provide solutions for computing the optimal placement that maximizes the system capacity for both transmission rate models in different network settings.

In a general scenario, there is an AP located at the origin, and MHs are distributed in the coverage area of the BSS with some probability density function $h(x, y)$. There is a fixed number of road segments, denoted by the set $\Lambda=$ $\{1,2, \ldots, M\}$, that the service provider is allow to install EPs. For each road segment, $i$, the service provider allocates $N_{i}$, where $i \in \Lambda$ and $N_{i} \geq 1$, EPs to be installed. Moreover, the starting and ending points of each road segment are clearly defined, and the length a road segment, $i$, is $d_{\text {max }}^{i}$. Furthermore, the decision variable, $d_{N_{i}, j}^{i}$, represents the location of the $j^{t h}$ out of $N_{i}$ EPs on the $i^{t h}$ road segment. Then, the EP locations can be represented by the vector $\underline{d}=$ $\left[d_{N_{1}, 1}^{1}, \ldots, d_{N_{1}, N_{1}}^{1}, \ldots, d_{N_{M}, 1}^{M}, \ldots, d_{N_{M}, N_{M}}^{M}\right]$. Fig. 4 illustrates a simple example of EP placement on two road segments.

If a $\mathrm{MH}$ at $(x, y)$ does not use an EP, its expected packet transaction time is

$T_{\text {noep }}(x, y)=T\left(\sqrt{x^{2}+y^{2}}, P_{a}, x_{d}\right)+T\left(\sqrt{x^{2}+y^{2}}, P_{m}, x_{u}\right)$,

where $T$ can be either $T_{s}$ or $T_{g}$, depending on the transmission rate model used. Now, suppose the $\mathrm{MH}$ decides to use an EP on road segment $i$ to relay its packet. According to Eqn. 


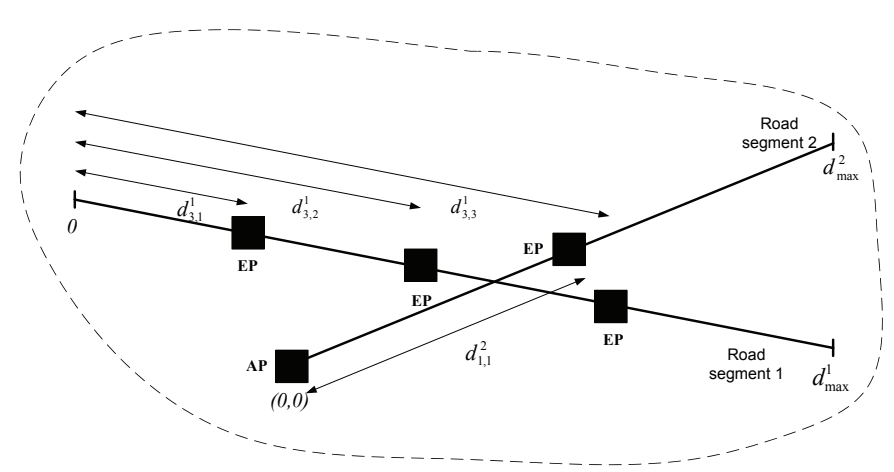

Fig. 4. A simple network configuration.

(3), the expected packet transaction time is $T_{e p}(x, y, d)$. In an environment with multiple road segments, we denote the expected packet transaction time by $T_{e p}^{i}(x, y, d)$, where the superscript $i$ represents road segment $i$.

By using an EP, the same data has to be transmitted twice. As a result, the EP may or may not be beneficial to a $\mathrm{MH}$. Therefore, before a mobile host transmits, it has to decide whether to use the EP to facilitate its communication with the $\mathrm{AP}$, or communicate with the AP directly. A MH will use an EP to facilitate its communication with the AP only if such usage results in a smaller expected packet transaction time, i.e., if $T_{e p}^{i}(d, x, y)<T_{\text {noep }}(x, y)$. Since the MHs are distributed in the coverage area $A$ probabilistically with a probability density function $h(x, y)$, the network expected packet transaction time is

$$
\begin{aligned}
& \overline{T_{e p}(\underline{d})}= \\
& \int_{A} h(x, y) \min \left[T_{\text {noep }}(x, y), \min _{i \in \Lambda} \min _{j=1, \ldots, N_{i}} T_{e p}^{i}\left(x, y, d_{N_{i}, j}^{i}\right)\right] d
\end{aligned}
$$

Using (16), we can cast the optimal EP placement problem as the following optimization problem.

$$
\text { Objective: } \min _{\underline{d}} \overline{T_{e p}(\underline{d})} \text {. }
$$

Clearly, this problem is difficult to solve directly. In the following subsections, we first propose a general solution formulation, which is in a form that can be handle by commercial non-linear optimization software, for the EP placement problem in a general network scenario. Then, we present an efficient algorithm that solves the EP placement problem in a single road segment scenario.

\section{A. General Solution Formulation}

To solve the EP placement problem in a general network scenario, we calculate the value of (16) by discretizing the network into a large but finite number of areas, where a mobile host is located at each area with a certain probability. Then, the integral in (16) can be interpreted as a Riemann sum. As shown in Fig. 5, we can divide the entire network into $L^{2}$ equally sized cells ${ }^{6}$, and the size of each cell is $\Delta^{2}$. A MH is located in each cell with a certain probability, and if a $\mathrm{MH}$

\footnotetext{
${ }^{6} \mathrm{To}$ maintain symmetry, $L$ is divisible by 2 .
}

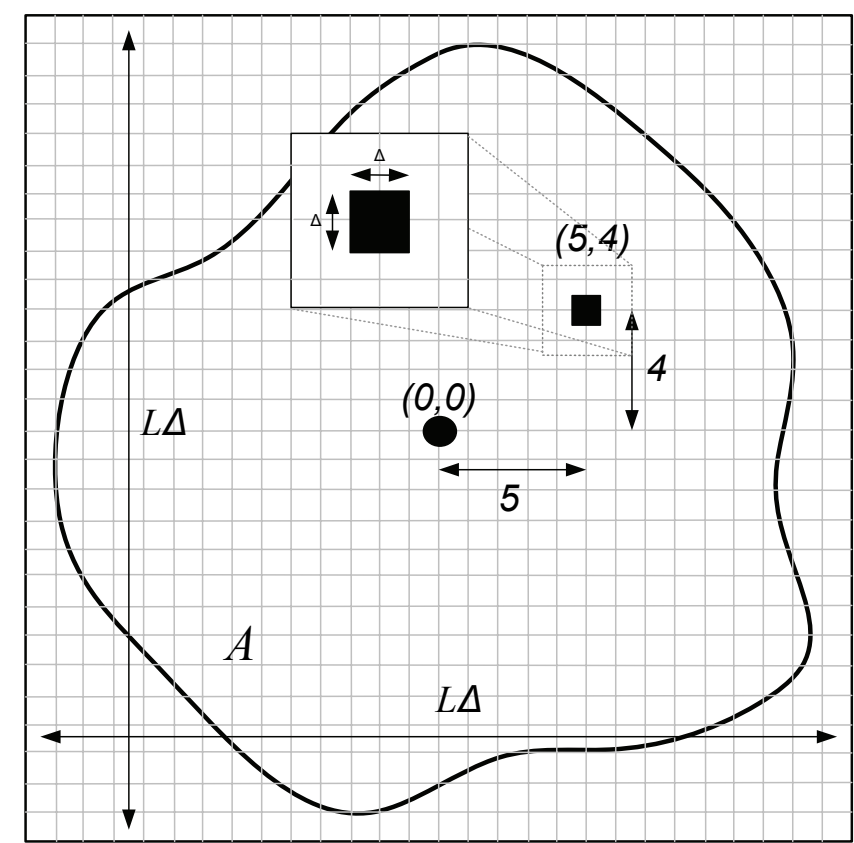

Fig. 5. Discretization of the network.

occupies a cell, we assume that it is located in the middle of the cell. Then, each MH can be uniquely identified by its Cartesian coordinate, and the AP is located at the origin. For example, the selected site in Fig. 5 is indexed by $(5,4)$. In this approximated reformulation, an area occupies by a cell is represented by a single $\mathrm{MH}$ located in the center of the cell. Thus, the probability that a $\mathrm{MH}$ at $(x, y)$ is present can be $A$.calculated by

$$
h_{x, y}=\int_{\Delta(x-0.5)}^{\Delta(x+0.5)} \int_{\Delta(y-0.5)}^{\Delta(y+0.5)} h(x, y) d x d y
$$

Then, (16) can be approximated as

$$
\begin{array}{r}
\overline{T_{e p}(\underline{d})}=\sum_{x=-\frac{L}{2}} \sum_{y=-\frac{L}{2}}^{\frac{L}{2}} h_{x, y} \min \left[T_{\text {noep }}(x \Delta, y \Delta)\right. \\
\left.\min _{i \in \Lambda} \min _{j=1, \ldots, N_{i}} T_{e p}^{i}\left(x \Delta, y \Delta, d_{N_{i}, j}^{i}\right)\right] .
\end{array}
$$

To facilitate the minimization of $\overline{T_{e p}(\underline{d})}$, we define the following decision variables, $\mathbf{Y}$, such that

$Y_{(x, y),(i, j)}= \begin{cases}1 & \begin{array}{l}\text { if the } \mathrm{MH}(x, y) \text { uses the } y^{t h} \mathrm{EP} \\ 0\end{array} \\ \text { on the } i^{t h} \text { road segment. } & \text { otherwise. }\end{cases}$

Furthermore, we set $Y_{(x, y),(0,0)}=1$ only if the $\mathrm{MH}$ at $(x, y)$ uses the AP directly. 


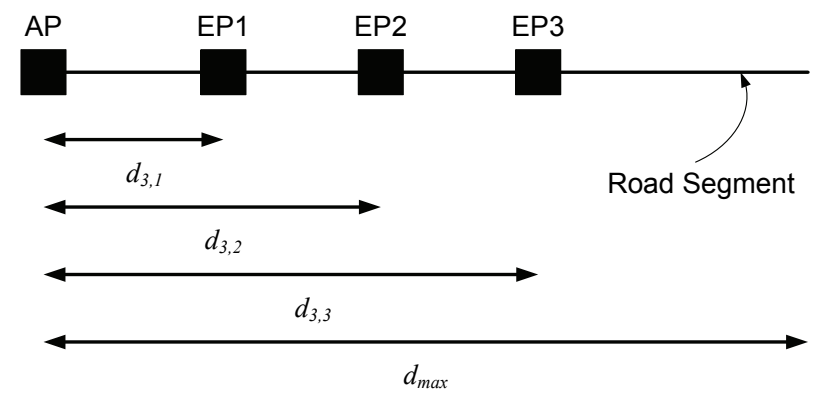

Fig. 6. Multi-user one-dimensional WLAN with multiple EPs.

Therefore, (17) can be reformulated as

$$
\begin{aligned}
& \min _{\mathbf{Y}, \underline{d}}: \sum_{x=-\frac{L}{2}}^{\frac{L}{2}} \sum_{y=-\frac{L}{2}}^{\frac{L}{2}} h_{x, y}\left[T_{n o e p}(x \Delta, y \Delta) Y_{(x, y),(0,0)}\right. \\
&\left.+\sum_{i \in \Lambda} \sum_{j=1}^{N_{i}} T_{e p}^{i}\left(x \Delta, y \Delta, d_{N_{i}, j}^{i}\right) Y_{(x, y),(i, j)}\right](21) \\
& \text { s.t. } \quad Y_{(x, y),(0,0)}+\sum_{i \in \Lambda} \sum_{j=1}^{N_{i}} Y_{(x, y),(i, j)}=1 \quad \forall(x, y)(22)
\end{aligned}
$$

Objective (21) minimizes the sum of the network expected packet transaction time. Constraint (22) requires each $\mathrm{MH}$ to be assigned to exactly one EP or the AP.

Because of the binary constraints (20), the formulation above is a non-linear integer programming problem, which is difficult to solve. However, we can show that if we replace the binary constraints (20) with its equivalent linear constraints, i.e., $0 \leq Y_{(x, y),(i, j)} \leq 1$, the resulting problem is a nonlinear problem with linear constraints. We can also show that when the relaxed problem is solved to its optimality, $\mathbf{Y}$ must be binary. The proofs of the above arguments can be found in the Appendix. The relaxed problem is in the form that can be handled by commercial non-linear optimization solvers such as MOSEK and CPLEX. In addition, our earlier work [31] presented an approximate optimization formulation for a more general framework similar to the above problem. In the next subsection, we discuss a network scenario where we can solve the EP placement problem analytically with an efficient algorithm based on recursion.

\section{B. Analytical Solution for a Single Road Segment}

In a common scenario, where the AP, EPs and MHs are located on one road segment, the optimal placement of the EPs can be calculated analytically. In this scenario, MHs are distributed on the road segment under consideration with a probability density function $h(l)$, and the length of this road segment is $d_{\max }$, and $N$ EPs are to be installed on the same road segment. Fig. 6 shows a simple example, where there are 3 EPs available on the right hand side of the AP. ${ }^{7}$ Since the network under consideration is one-dimensional, $T_{\text {noep }}(x, y)$ and $T_{e p}^{i}(x, y, d)$ become $T_{n o e p}(x, 0)$ and $T_{e p}^{1}(x, 0, d)$ respectively. For notation simplifications, we denote $T_{\text {noep }}(x, 0)$ by

\footnotetext{
${ }^{7}$ Because of symmetry, we can ignore the left hand side of the system in our discussion.
}

$T_{\text {noep }}(x)$ and $T_{e p}^{1}(x, 0, d)$ by $T_{e p}(x, d)$. Thus, Eqn. (16) can be re-written as the following.

$\overline{T_{e p}(\underline{d})}=\int_{0+\epsilon}^{d_{\max }} h(l) \min \left[T_{n o e p}(l), \min _{j=1, \ldots, N} T_{e p}\left(l, d_{N, j}\right)\right] d l$,

where $\epsilon>0$ is small and $\underline{d}=\left[d_{N, 1}, \ldots, d_{N, N}\right]$. In this subsection, we show that the optimization problem of minimizing the modified $\overline{T_{e p}(\underline{d})}$, i.e., Eqn (23) with respect to $\underline{d}$ can be solved analytically.

We first show that each EP is responsible to serve a continuous segment of the network. Then, the minimization function inside the integral of (23) can be removed by taking the integration segment by segment. This simplifies the analysis and eventually facilitates the development of a computationally feasible algorithm that solves the optimization problem.

Theorem 1: Given $T(l, P, z)$, where $T$ represents either $T_{s}$ or $T_{g}$, is an increasing and convex function of $l$ within the region of interest, for any two $\mathrm{EPs}, \mathrm{EP}_{i}$ and $\mathrm{EP}_{j}$, which are located at $d_{i}$ and $d_{j}$ respectively, where $i<j$ and $d_{i}<d_{j}$, there exists an $l^{*}>\frac{d_{i}+d_{j}}{2}$ such that $\forall l>l^{*}, T_{e p}\left(l, d_{i}\right)>$ $T_{e p}\left(l, d_{j}\right)$ and $\forall l<l^{*}, T_{e p}\left(l, d_{i}\right)<T_{e p}\left(l, d_{j}\right)$.

Proof: According to Eqn.(3), in this one-dimensional scenario,

$$
\begin{aligned}
T_{e p}(l, d)= & T\left(d, P_{a}, z_{d}\right)+T\left(|l-d|, P_{e}, z_{d}\right)+ \\
& T\left(|l-d|, P_{m}, z_{u}\right)+T\left(d, P_{e}, z_{u}\right) .
\end{aligned}
$$

In this proof, we will analyze the relationship between $T_{e p}\left(l, d_{i}\right)$ and $T_{e p}\left(l, d_{j}\right)$ with respect to $l$ at three different regions of the network:

1) $l<\frac{d_{i}+d_{j}}{2}$ : Since $T(l, P, z)$ is an increasing function of $l$, the following four statements are always true.

- $T\left(d_{i}, P_{a}, z_{d}\right)<T\left(d_{j}, P_{a}, z_{d}\right)$

- $T\left(d_{i}, P_{e}, z_{u}\right)<T\left(d_{j}, P_{e}, z_{u}\right)$

- $T\left(\left|l-d_{i}\right|, P_{e}, z_{d}\right)<T\left(\left|l-d_{j}\right|, P_{e}, z_{d}\right)$

- $T\left(\left|l-d_{i}\right|, P_{m}, z_{u}\right)<T\left(\left|l-d_{j}\right|, P_{m}, z_{u}\right)$

Therefore, $T_{e p}\left(l, d_{i}\right)<T_{e p}\left(l, d_{j}\right)$.

2) $\frac{d_{i}+d_{j}}{2}<l<d_{j}$ : From the above, we know that $T_{e p}\left(\frac{d_{i}+d_{j}}{2}, d_{i}\right)<T_{e p}\left(\frac{d_{i}+d_{j}}{2}, d_{j}\right)$. Moreover, in this region, $T_{e p}\left(l, d_{i}\right)$ is an increasing function of $l$, and $T_{e p}\left(l, d_{j}\right)$ is a decreasing function of $l$. As a result, depends on the values of $T_{e p}\left(d_{j}, d_{i}\right)$ and $T_{e p}\left(d_{j}, d_{j}\right), T_{e p}\left(l, d_{i}\right)$ and $T_{e p}\left(l, d_{j}\right)$ may or may not intersect. This gives us two cases.

First, if $T_{e p}\left(d_{j}, d_{i}\right)<T_{e p}\left(d_{j}, d_{j}\right), T_{e p}\left(l, d_{i}\right)$ and $T_{e p}\left(l, d_{j}\right)$ will not intersect in this region. So, $\forall l<d_{j}, T_{e p}\left(l, d_{i}\right)<$ $T_{e p}\left(l, d_{j}\right)$.

Second, if $T_{e p}\left(d_{j}, d_{i}\right)>T_{e p}\left(d_{j}, d_{j}\right), T_{e p}\left(l, d_{i}\right)$ and $T_{e p}\left(l, d_{j}\right)$ will intersect once in this region. This means that there exist an $l^{*}$, where $\frac{d_{i}+d_{j}}{2}<l^{*}<d_{j}$, such that $\forall l<$ $l^{*}, T_{e p}\left(l, d_{i}\right)<T_{e p}\left(l, d_{j}\right)$ and $\forall l \in\left(l^{*}, d_{j}\right), T_{e p}\left(l, d_{i}\right)>$ $T_{e p}\left(l, d_{j}\right)$.

3) $l>d_{j}$ : For $l>d_{j}$, both $T_{e p}\left(l, d_{i}\right)$ and $T_{e p}\left(l, d_{j}\right)$ are increasing functions of $l$. Since $T(l, P, z)$ is a convex function of $l$ in this region, $T\left(\left|l-d_{i}\right|, P, z\right)$ increases at a faster rate than $T\left(\left|l-d_{j}\right|, P, z\right)$. Consequently, $T_{e p}\left(l, d_{i}\right)$ increases in a faster rate than $T_{e p}\left(l, d_{j}\right)$ in this region. As a result, when $T_{e p}\left(d_{j}, d_{i}\right)<T_{e p}\left(d_{j}, d_{j}\right), T_{e p}\left(l, d_{i}\right)$ and 


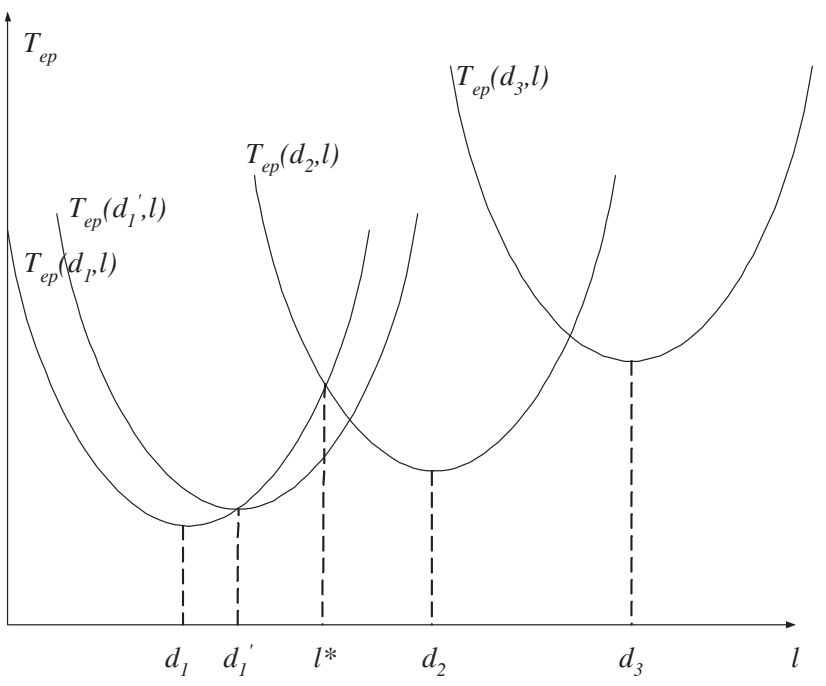

Fig. 7. Graphical illustration of packet transaction time at different locations.

$T_{e p}\left(l, d_{j}\right)$ will intersect at some point $l^{*}>d_{j}$, and $\forall l<l^{*}$, $T_{e p}\left(l, d_{i}\right)<T_{e p}\left(l, d_{j}\right)$, and $\forall l>l^{*}, T_{e p}\left(l, d_{i}\right)>T_{e p}\left(l, d_{j}\right)$. When $T_{e p}\left(d_{j}, d_{i}\right)>T_{e p}\left(d_{j}, d_{j}\right), T_{e p}\left(l, d_{i}\right)$ and $T_{e p}\left(l, d_{j}\right)$ will not intersect again in this region $\left(l>d_{j}\right)$.

In short, in all cases, there exists an $l^{*}>\frac{d_{i}+d_{j}}{2}$ that divides the network into two continuous segments, and in each segment, one EP is preferred over the other.

Theorem 1 shows that, for every two adjacent EPs, $\mathrm{EP}_{i}$ and $\mathrm{EP}_{i+1}$, there exist only one $l^{*}$ that divides the EP neighborhood into two continuous service regions. We can interpret $l^{*}$ as the upper boundary of $\mathrm{EP}_{i}$ service region and the lower boundary of $\mathrm{EP}_{i+1}$ service region. Therefore, with multiple EPs, the network can be partitioned into several continuous service regions, and each service region is served by a dedicated EP.

To explain the implication of Theorem 1 graphically, let us consider the following. According to Eqn. (24), for constant values of $P_{a}, P_{e}, P_{m}, z_{u}$ and $z_{d}, T_{e p}(l, d)=T\left(d, P_{a}, z_{d}\right)+$ $T\left(|l-d|, P_{e}, z_{d}\right)+P\left(|l-d|, P_{m}, z_{u}\right)+P\left(d, P_{e}, z_{u}\right)$. Thus, for fixed $d_{1}<d_{2}<d_{3}, T_{e p}\left(l, d_{1}\right), T_{e p}\left(l, d_{2}\right)$, and $T_{e p}\left(l, d_{3}\right)$, are vertically and horizontally shifted version of $l$ of each other ${ }^{8}$, as shown in Fig. 7. For a fixed value of $d_{2}$ and for all values of $d_{1}<d_{2}$, the location of $\mathrm{EP}_{3}$, i.e., $d_{3}>d_{2}$, will not affect the location of the upper boundary of $\mathrm{EP}_{1}$ service region, $l^{*}$. Thus, $l^{*}$ is only dependent on the locations of $\mathrm{EP}_{1}$ and $\mathrm{EP}_{2}$, but not $\mathrm{EP}_{3}$. The same argument applies to the location of the lower boundary of an EP service region.

Since $l^{*}$ is a function of $d_{i}$ and $d_{j}$, let us rename $l^{*}$ to $f\left(d_{i}, d_{j}\right)$. For all $l<f\left(d_{i}, d_{j}\right)$, the MHs are serviced by $\mathrm{EP}_{i}$ (or not serviced by any EP if the MH is very close to the AP), while the rest of the network is serviced by $\mathrm{EP}_{j}$. Similarly, if another EP is placed at $d_{k}$, where $d_{j}<d_{k}$, there exist a $f\left(d_{j}, d_{k}\right)$ that divide the service regions of $\mathrm{EP}_{j}$ and $\mathrm{EP}_{k}$. As a

${ }^{8}$ Note that, for constant values of $P$ and $z, T(l, P, z)$ is assumed to be a increasing convex function of $l$. The region of convexity of $T_{s}(l, P, z)$ and $T_{g}(l, P, z)$ and their effects on the proposed optimization algorithm will be discussed in the Appendix.

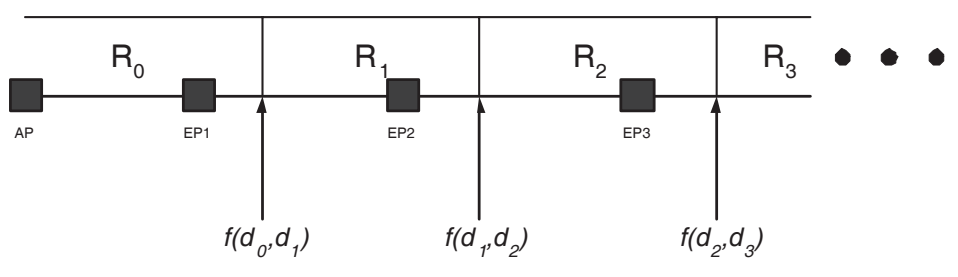

Fig. 8. Continuous service regions for different EPs.

result, the service region of $\mathrm{EP}_{j}$ is from $f\left(d_{i}, d_{j}\right)$ to $f\left(d_{j}, d_{k}\right)$. Fig. 8 describes a simple example, which illustrates the service regions corresponding to the network in Fig. 6. $R_{1}, R_{2}, R_{3}$ are the service regions of EP1, EP2 and EP3 respectively. Notice that, if an EP is placed at the AP, $d_{0}=0$, the system is unchanged. Thus, $R_{0}$ denotes the service region serviced directly by the AP. We denote the border between regions $R_{i}$ and $R_{i+1}$ as $f\left(d_{i}, d_{i+1}\right)$.

Then, for a given EP placement $\underline{d}=\left[d_{N, 1}, \ldots, d_{N, N}\right]$, and letting $d_{N, 0}=0,(23)$ can be rewritten as

$$
\begin{aligned}
& \overline{T_{e p}(\underline{d})} \\
&=\int_{0+\epsilon}^{d_{\max }} h(l) \min \left[T_{\text {noep }}(l), T_{e p}\left(l, d_{N, 1}\right), \ldots, T_{e p}\left(l, d_{N, N}\right)\right] d l \\
&=\int_{0+\epsilon}^{f\left(d_{N, 0}, d_{N, 1}\right)} h(l) T_{\text {noep }}(l) d l+ \\
& \sum_{i=1}^{N-1} \int_{f\left(d_{N, i-1}, d_{N, i}\right)}^{f\left(d_{N, i}, d_{N, i+1}\right)} h(l) T_{e p}\left(l, d_{N, i}\right) d l+ \\
& \int_{f\left(d_{N, N-1}, d_{N, N}\right)}^{d_{\text {max }}} h(l) T_{e p}\left(l, d_{N, N}\right) d l .
\end{aligned}
$$

Since it is not possible to obtained a closed form for $f\left(d_{N, i-1}, d_{N, i}\right)$, the gradient of this objective is still hard to compute. However, by using (25), we can obtain some characteristics about the gradient of $\overline{T_{e p}(\underline{d})}$. We simplify (25) further in the following. Let

$$
\begin{aligned}
\operatorname{TIME} E_{\text {noep }}(l) & =\int h(l) T_{\text {noep }}(l) d l \\
T I M E_{e p}\left(l, d_{N, i}\right) & =\int h(l) T_{e p}\left(l, d_{N, i}\right) d l .
\end{aligned}
$$

Then

$\overline{T_{e p}(\underline{d})}=$

$\left[T I M E_{\text {noep }}\left(f\left(d_{N, 0}, d_{N, 1}\right)\right)-T I M E_{\text {noep }}(0+\epsilon)\right]+$

$\left[\sum_{i=1}^{N-1} T I M E_{e p}\left(f\left(d_{N, i}, d_{N, i+1}\right), d_{N, i}\right)\right.$

$\left.-\sum_{i=1}^{N-1} T I M E_{e p}\left(f\left(d_{N, i-1}, d_{N, i}\right), d_{N, i}\right)\right]+$

$\left[T I M E_{e p}\left(d_{\max }, d_{N, N}\right)-T I M E_{e p}\left(f\left(d_{N, N-1}, d_{N, N}\right), d_{N, N}\right)\right]$.

From (26), it is clear that $\frac{\partial \overline{T_{e p}(d)}}{\partial d_{N, 1}}$ is a function of $d_{N, 1}$ and $d_{N, 2}$ only, $\frac{\partial \overline{T_{e p}(\underline{d})}}{\partial d_{N, i}}$ is a function of $d_{N, i-1}, d_{N, i}$, and $d_{N, i+1}$ only, for $1<i<N$, while $\frac{\partial \overline{T_{e p}(\underline{d})}}{\partial d_{N, N}}$ is a function 
of $d_{N, N-1}$ and $d_{N, N}$ only. This observation is summarized in the following.

$$
\begin{aligned}
& \frac{\partial \overline{T_{e p}(\underline{d})}}{\partial d_{N, 1}}=\xi_{1}\left(d_{N, 1}, d_{N, 2}\right) \\
& \frac{\partial \overline{T_{e p}(\underline{d})}}{\partial d_{N, i}}=\xi_{i}\left(d_{N, i-1}, d_{N, i}, d_{N, i+1}\right), \text { for } 1<i<N \\
& \frac{\partial \overline{T_{e p}(\underline{d})}}{\partial d_{N, N}}=\xi_{N}\left(d_{N, N-1}, d_{N, N}\right)
\end{aligned}
$$

The important implication from this is that, given $d_{N, i-1}$ and $d_{N, i+1}$, the rate of change of $\overline{T_{e p}(\underline{d})}$ with respect to $d_{N, i}$ does not depend on the position of the other EPs. For a global minimum and all other critical points, all $\frac{\partial \overline{T_{e p}(\underline{d})}}{\partial d_{N, i}} \forall i$ s.t. $1 \leq$ $i \leq N$, i.e., the gradient of the objective function, must equal zero. Thus, an algorithm which is able to find all the critical points, i.e., $\underline{d}$ which results in zero gradient, of the optimization objective is desired. In the next subsection, we present a simple algorithm to solve the optimization problem using the above result.

\section{Optimization Algorithm}

The goal of the algorithm is to find all the critical points of the optimization objective function (23). We discretized the network space into $n$ small equally spaced portions, each of which is considered to be a possible location of an EP. Each possible location is indexed from 1 to $n$ according to their distance from the AP. Then, the objective is to choose $N$ out of $n$ possible locations to place these EPs such that the expected throughput capacity of the network is maximized.

Since the optimal location of an EP only depends on the locations of its adjacent EPs, we can treat this problem as a sequence of 2-EP and 3-EP placement problems. First, we can obtain the full characteristics of the 3-EP and 2-EP networks. Then this information can be used recursively to determine the optimal placement of the EPs in an $N$-EP network.

The following three sets of values for the 2-EP and 3-EP networks are first computed numerically.

$$
\begin{aligned}
a(x)= & \text { Optimal } d_{2,1} \text { given } d_{2,2}=x \\
b(x, y)= & \text { Optimal } d_{3,2} \text { given } d_{3,1}=x \text { and } d_{3,3}=y \\
c(x)= & \text { Optimal } d_{2,2} \text { given } d_{2,1}=x \\
& \forall x, y \in[1, n]
\end{aligned}
$$

To illustrate how the proposed algorithm works, let us consider the following 4 EP placement example. For an optimal solution, $\underline{d}=\left[d_{4,1}, d_{4,2}, d_{4,3}, d_{4,4}\right]$, the gradient must be zero. In other words, $\xi_{1}\left(d_{4,1}, d_{4,2}\right)=0, \xi_{2}\left(d_{4,1}, d_{4,2}, d_{4,3}\right)=0$, $\xi_{3}\left(d_{4,2}, d_{4,3}, d_{4,4}\right)=0$, and $\xi_{4}\left(d_{4,3}, d_{4,4}\right)=0$. This implies that $d_{4,1}=a\left(d_{4,2}\right), d_{4,2}=b\left(d_{4,1}, d_{4,3}\right), d_{4,3}=b\left(d_{4,2}, d_{4,4}\right)$, and $d_{4,4}=c\left(d_{4,3}\right)$. The purpose of our proposed algorithm is to generate points which satisfy these properties recursively. It is carried out as follows.

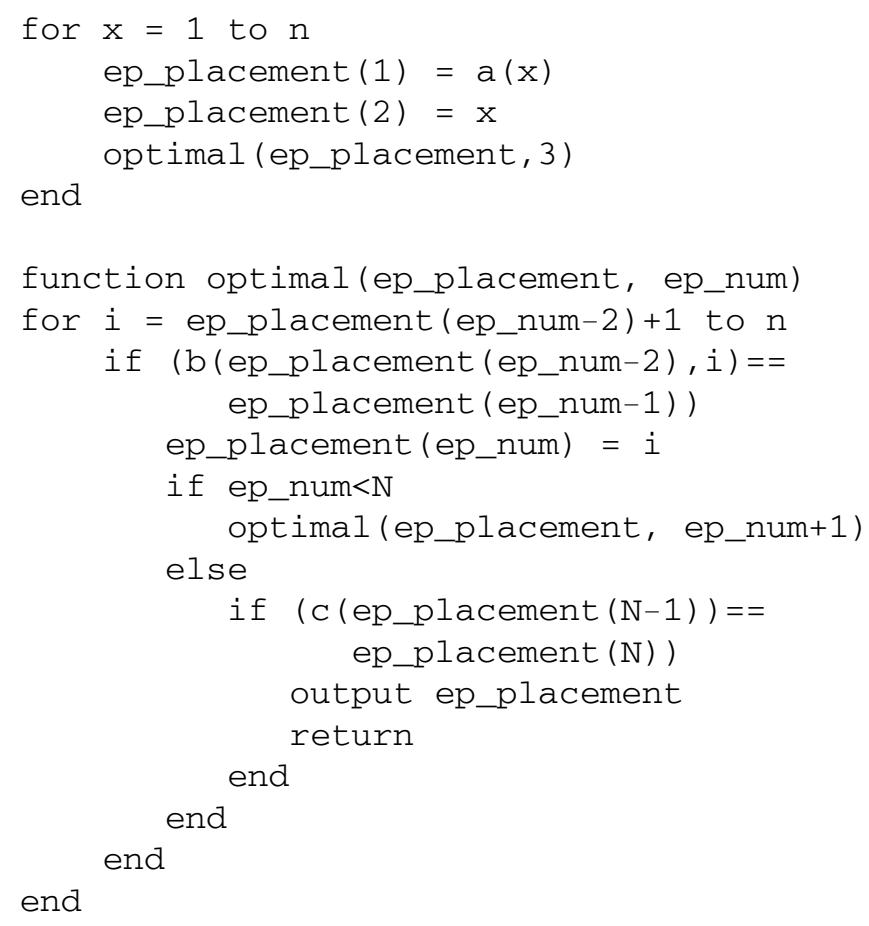

In this algorithm, ep_placement is an array with $N$ elements which represent the optimal EP locations. The function optimal is an recursive function that is used to search for all the critical points. If the algorithm returns more than one output, we evaluate all of them and choose the global minimum.

\section{Numerical AnAlysis}

In this section, we discuss the benefit of the optimally placed extension points with respect to different channel models and system parameters. Both of the two channel models described in Section III-C.1 and Section III-C. 2 were used. In addition, the system that we investigated has four system parameters: roll off factor $(\alpha)$, proportion of downlink data $(\beta)$, power of the AP and EP over power of $\mathrm{MH} \operatorname{ratio}^{9}\left(P_{a e} / P_{m}\right)$, and the number of EPs $(N)$. By using a particular channel model, for each set of parameters, there is an optimal placement of EPs and two capacities: $C_{n o e p}$ and $C_{e p}$, where $C_{n o e p}$ represents the throughput capacity of the network without extension points, while $C_{e p}$ represents the throughput capacity of the network with the extension points optimally placed. The throughput capacity of the network is defined in (2). Thus, $C_{n o e p}$ and $C_{e p}$ are defined as follows:

$$
C_{\text {noep }}=\frac{z}{\overline{T_{\text {noep }}}} \quad, \quad C_{e p}=\frac{z}{\overline{T_{e p}\left(\underline{\left.d^{*}\right)}\right.}},
$$

where $d^{*}$ represents the optimal location(s) for the EPs. We define the performance gain of the network as

$$
\text { Gain }=\frac{C_{e p}-C_{n o e p}}{C_{n o e p}} .
$$

\footnotetext{
${ }^{9}$ In our numerical analysis, we let the AP and EP to have the same transmitter reference power, and it is denoted by $P_{a e}$.
} 


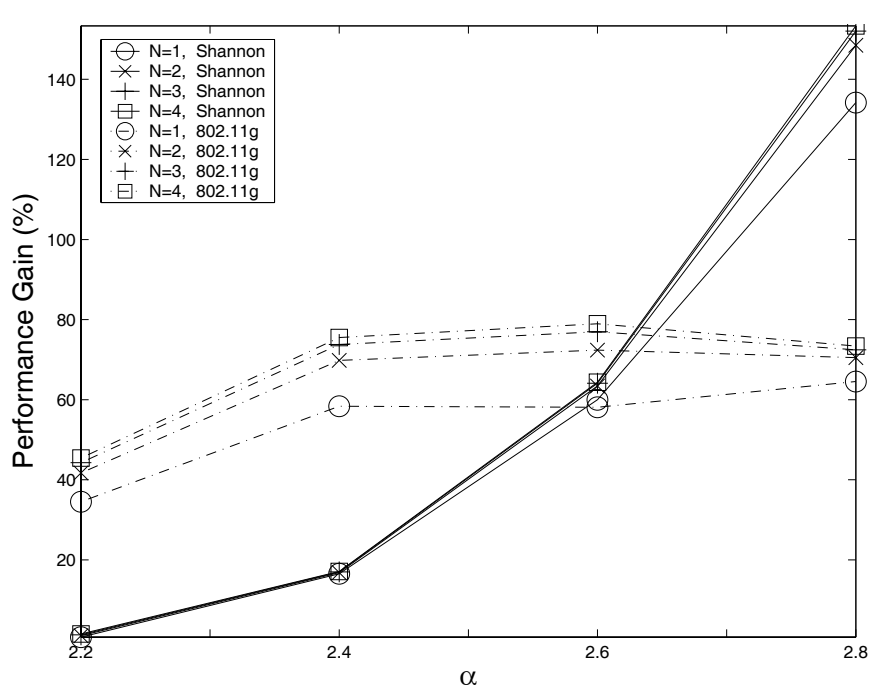

Fig. 9. Optimal performance gain with respect to different roll-off factors under Shannon point-to-point channel model and $802.11 \mathrm{~g}$ bit rates with Rayleigh fading channel model.

For a particular set of system parameters, two different optimal EP placements can be computed under the two channel models. Let $\underline{d_{S}}=\left(d_{N, 1}^{S}, \ldots, d_{N, N}^{S}\right)^{T}$ and $\underline{d_{G}}=$ $\left(d_{N, 1}^{G}, \ldots, d_{N, N}^{G}\right)^{T}$ be the optimal EP placements obtained by using the Shannon point-to-point channel model and IEEE 802.11g model under Rayleigh fading, respectively. The average placement difference between the two channel models is defined as

$$
D=\frac{1}{N} \sum_{i=1}^{N} \frac{\left|d_{N, i}^{S}-d_{N, i}^{G}\right|}{d_{N, i}^{S}} .
$$

In the following, the relationship among the four system parameters with respect to the optimal EP placement and performance gain with different channel models will be discussed.

\section{A. Roll Off Factor}

We study the effect of the roll off factor $(\alpha)$ and the number of EPs $(N)$ with both channel models in Fig. 9, Fig. 10 and Fig. 11. Like a typical WLAN in an outdoor environment, the network provide a 400 meters coverage area. Both the AP and EP are equipped with a $10 \mathrm{dBm}$ transmitter, while the mobile hosts use a $5 \mathrm{dBm}$ transmitter. All transmitters and receivers have $2.2 \mathrm{dBi}$ antenna gain. The network occupies a $20 \mathrm{MHz}$ channel in the $2.4 \mathrm{GHz}$ spectrum, and a $-90 \mathrm{dBm}$ noise power is assumed anywhere in the network. The combined length of a uplink and a downlink packet is set to $2 \mathrm{k}$ bytes, and $70 \%$ of downlink traffic is assumed.

Fig. 9 and Fig. 10 show the performance gains and optimal EP placements with respect to different roll off factors respectively. By using the Shannon point-to-point channel model, when the roll off factor is low, the entire network can transmit at very high rates; thus, the beneficial effects of the EPs are small. As shown in Fig. 10, when the EPs are optimally placed, they are located very close to the edge of the network so that they can only help the small segments of the network which have the worst throughput. As a result, the beneficial effects

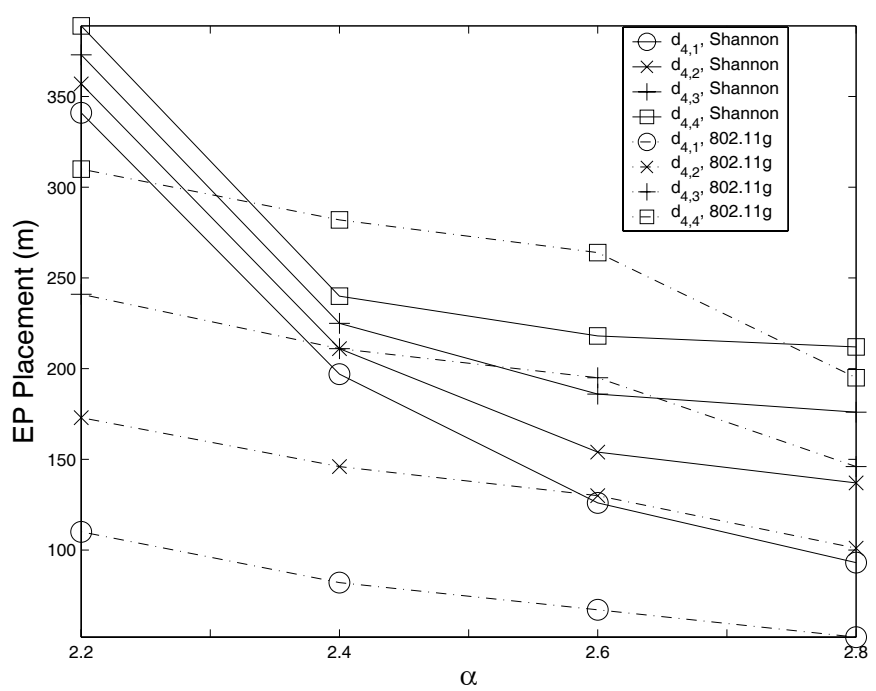

Fig. 10. Optimal placement of 4 EPs with respect to different roll-off factors under Shannon point-to-point channel model and $802.11 \mathrm{~g}$ bit rates with Rayleigh fading channel model.

of the EPs are very low, and thus, as shown in Fig. 9, the performance gain is very low. The roll off factor determines how fast the signal decades when it travels through a distance. Therefore, as the roll off factor increases, the beneficial effects of the EPs become more significant. For a high roll off factor, the optimally placed EPs are more spread out. This means each EP is assigned to help a longer segment of the network when they are optimally placed. Consequently, the performance gain increases as the roll off factor increases.

Under the IEEE 802.11g model with Rayleigh fading, some different trends are observed. As shown in Fig. 10, when the roll off factor is small, the optimally placed EPs are quite spread out. In contrast, when the roll off factor is large, the EPs are located closer to each other and to the AP. This implies that the distanced MHs receive less benefit from the EPs when the roll off factor is large. This result is counter intuitive since as the signal attenuates faster, the role of the EPs becomes more significant to the distanced HMs. Intuitively, the EPs are expected to be placed closer to the edge of the network. This counter intuitive result suggests that when the roll off factor is large, it is more beneficial to allocate more EPs to help MHs that are relatively closer to the AP. In other words, in high channel attenuation, the marginal benefit provided by the EPs to the distanced MHs are less than that to the MHs which are near the AP.

From Fig. 9, by using both channel models and when the roll off factor is small, the performance gain increases as the roll off factor increases. When the roll off factor is large, under 802.11g with the Rayleigh fading channel model, the performance gain decreases as the roll off factor increases. In high channel attenuation environment, it is very hard to maintain high throughput at the edge of the network. In other words, the EPs become less powerful in terms of throughput improvement in such high channel attenuation environment. Therefore, as the roll off factor increases, the EPs can only prevent the network throughput from degrading further, but 


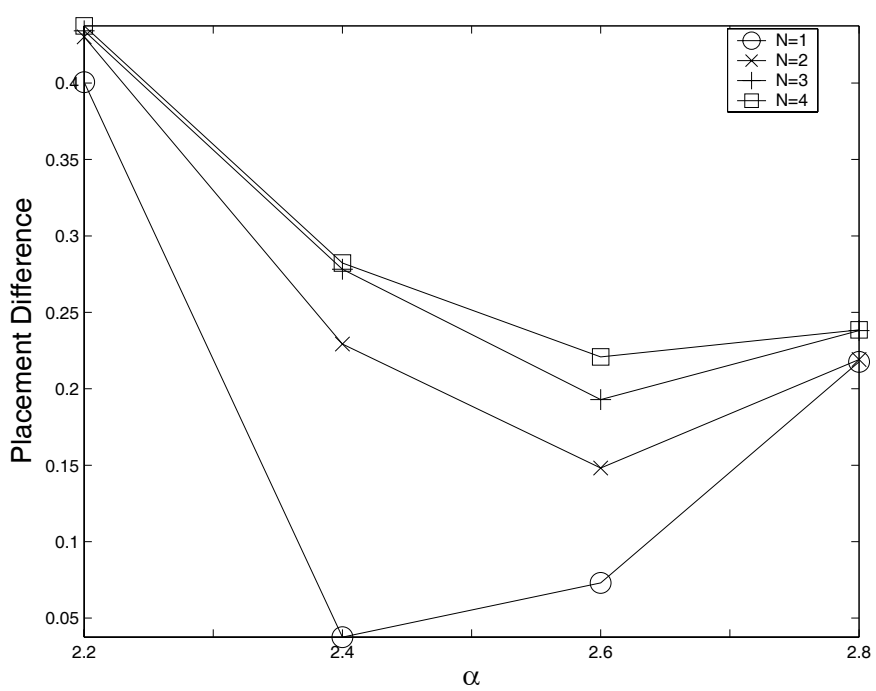

Fig. 11. Average placement difference between the two channel models with respect to different roll-off factors under Shannon Point-to-Point channel model and $802.11 \mathrm{~g}$ bit rates with Rayleigh fading channel model.

cannot provide high throughput improvement.

By using both channel models, the effect of diminishing return is observed as the number of EPs increases. Such phenomenon is more obvious under the Shannon point-topoint model or when the roll off factor is low. This observation suggests that when channel attenuation is small, a small number of EPs is sufficient to achieve reasonable performance gain, while in other cases, more EPs are needed to achieve higher performance gain.

In Fig. 11, we observed that the optimal placements obtained under the two channel models are quite different. In general, the placement different increases as the number of EPs increases. In this study, the Shannon point-to-point channel model is used to approximate the achievable bit rates of future WLANs. This results suggests that the optimal placements of the EPs will change if the AP and EPs are upgraded to a new WLAN standard which can achieve bit rates closer to the Shannon capacity bound.

\section{B. Proportion of Downlink and Uplink Traffic}

We study the effect of the proportion of downlink data $(\beta)$ and the number of EPs $(N)$ on an outdoor network with both channel models in Fig. 12, Fig. 13 and Fig. 14. The system parameters are the same as in Subsection V-A, except the roll off factor is set to 2.6. Moreover, the combined length of a uplink and a downlink packet is set to $2 \mathrm{k}$ bytes. I.e., the downlink and uplink packet lengths are $2 \beta \mathrm{k}$ bytes and $2(1-\beta) \mathrm{k}$ bytes respectively.

From Fig. 12, the performance gain increases as the proportion of uplink data, $(1-\beta)$, increases. This is, again, because the MH's transmitter has less power compared with that of the AP and EPs. As the amount of data needed to be transmitted by the MH's transmitter increases, the beneficial effect of the EP becomes more and more significant, which in turn result in higher performance gain.

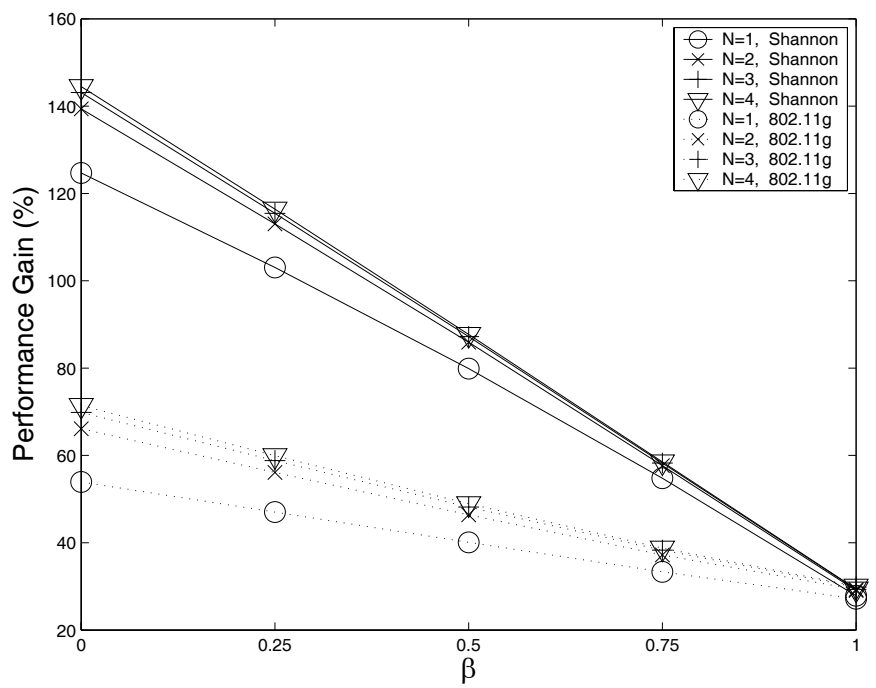

Fig. 12. Optimal performance gain with respect to proportion of downlink data under Shannon point-to-point channel model and 802.11g bit rates with Rayleigh fading channel model.

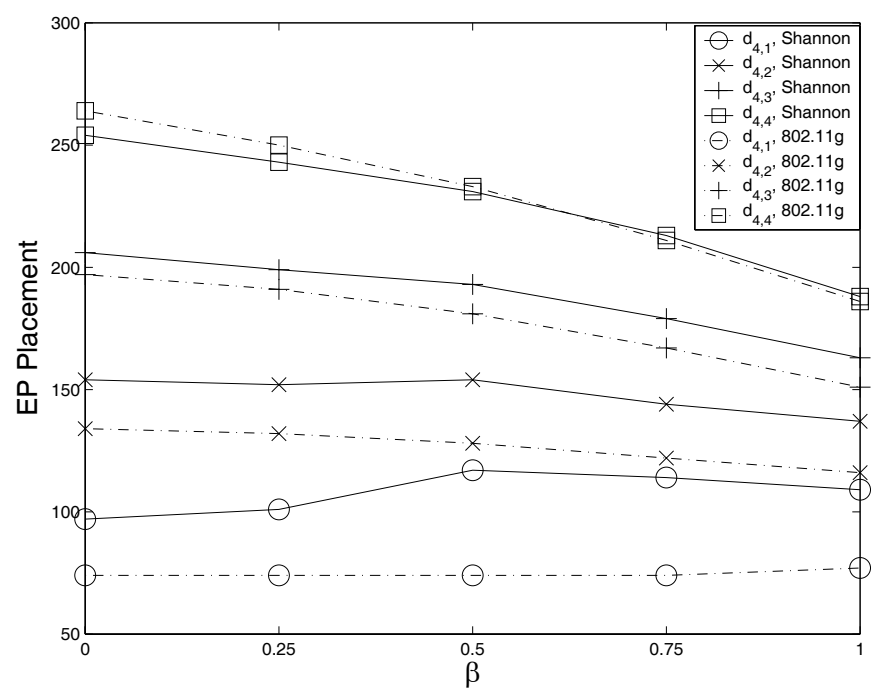

Fig. 13. Optimal placement of 4 EPs with respect to different proportion of downlink data under Shannon point-to-point channel model and $802.11 \mathrm{~g}$ bit rates with Rayleigh fading channel model.

From Fig. 13, by using both channel models, the optimally placed EPs are more spread out and further away from the AP when the proportion of downlink data, $\beta$, is low. This is because the MHs have less transmit power compared with that of AP and EP. When the proportion of downlink data is low, the MHs have more to transmit. When MHs have more to transmit, MHs that are located far away from the AP need more help compared with those located close to the AP. Therefore, in order to minimize the expected packet transaction time, the EPs should be placed further away from the AP. However, by using the $802.11 \mathrm{~g}$ bits rate with the Rayleigh fading channel model, the optimal placements of the EPs do not change significantly when the traffic characteristic changes slightly. This suggests that even the traffic varies slightly with time, the EPs found by the proposed algorithm 


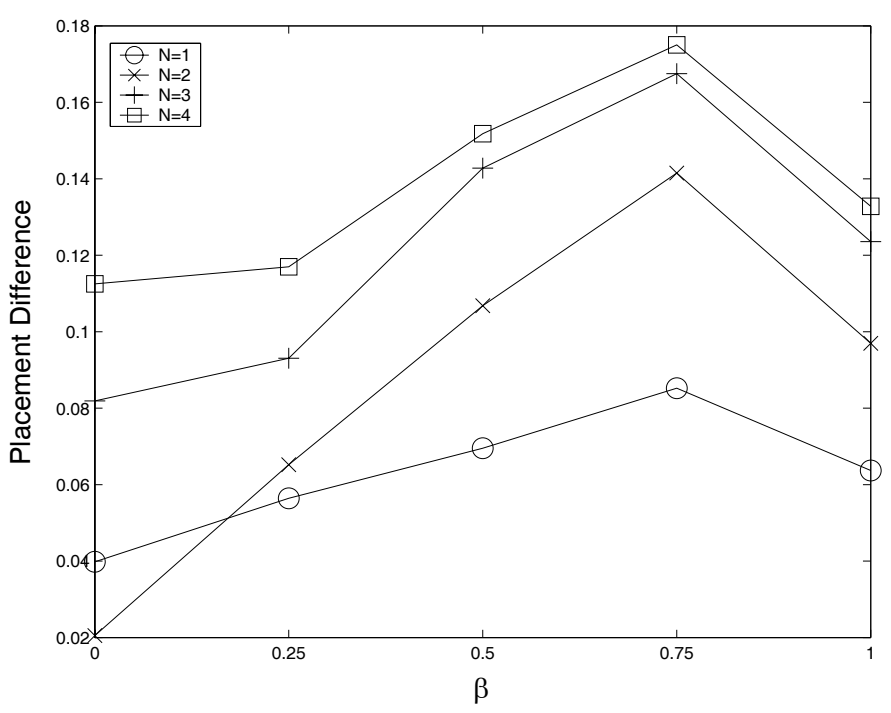

Fig. 14. Average placement difference between the two channel models with respect to different proportion of downlink data under Shannon Point-to-Point channel model and $802.11 \mathrm{~g}$ bit rates with Rayleigh fading channel model.

can always produce close to optimal results.

In Fig. 14, we studied the average placement difference between the two channel models. We observed that the difference of optimal placements obtained by the two channel models is small when the proportion of downlink data is small. As in the previous subsection, in most cases, the deviation between the two models increases when the number of EPs increases.

\section{AP/EP to MH Power Ratio}

We study the effect of the AP, EP to Mobile transmitter power ratio $\left(P_{a e} / P_{m}\right)$ and the number of EPs on an outdoor network with both channel models in Fig. 15, Fig. 16, and Fig. 17. The system parameters are the same as in Subsection V-A, except the system roll off factor is set to 2.4. Moreover, the AP's and EP's transmitter power are $15 \mathrm{dBm}$, while the MH's transmitter power varies depend on the $P_{a e} / P_{m}$ ratio.

From Fig. 15, the performance gain increases as the $\frac{P_{a e}}{P_{m}}$ ratio increases. The $\frac{P_{a e}}{P_{m}}$ ratio represents the difference of transmitter power between AP/EP and mobile hosts. When MH's transmitter power is low, the effect of the EPs becomes more significant. This is the reason why the performance gain is high when the mobile hosts have less powerful transmitters. Moreover, when the $\frac{P_{a e}}{P_{m}}$ ratio is high, the effect of diminishing return on larger number of EP is relatively low. Thus, with high power difference between the AP/EP and MHs, the marginal benefit of additional EP is relatively high. In other words, the installation of a larger number of EPs in such network can be justified.

From Fig. 16, the optimally placed EPs are more spread out when the $\frac{P_{a e}}{P_{m}}$ ratio is high. This is because the MHs need more help from the EPs when the MH's transmitter power is relatively low. Therefore, in order to minimize the expected packet transaction time, the EPs are more spread out so that each can serve a longer segment of the network.

In Fig. 17, we observed that the optimal placements obtained by the two channel models decreases as the $\frac{P_{a e}}{P_{m}}$ ratio

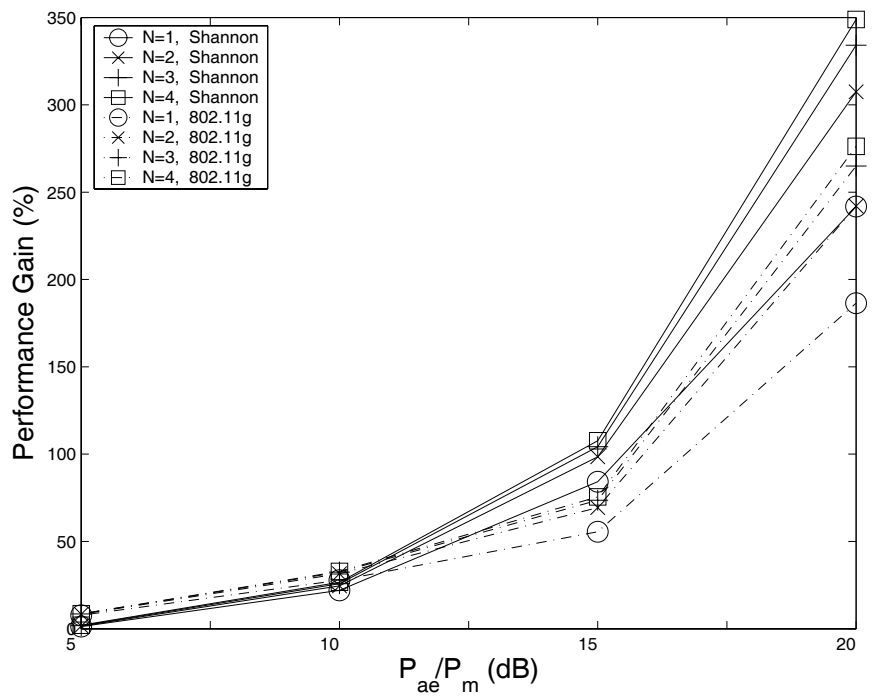

Fig. 15. Optimal capacity gain with respect to different $P_{a e} / P_{m}$ with Shannon point-to-point channel model and $802.11 \mathrm{~g}$ bit rates with Rayleigh fading channel model.

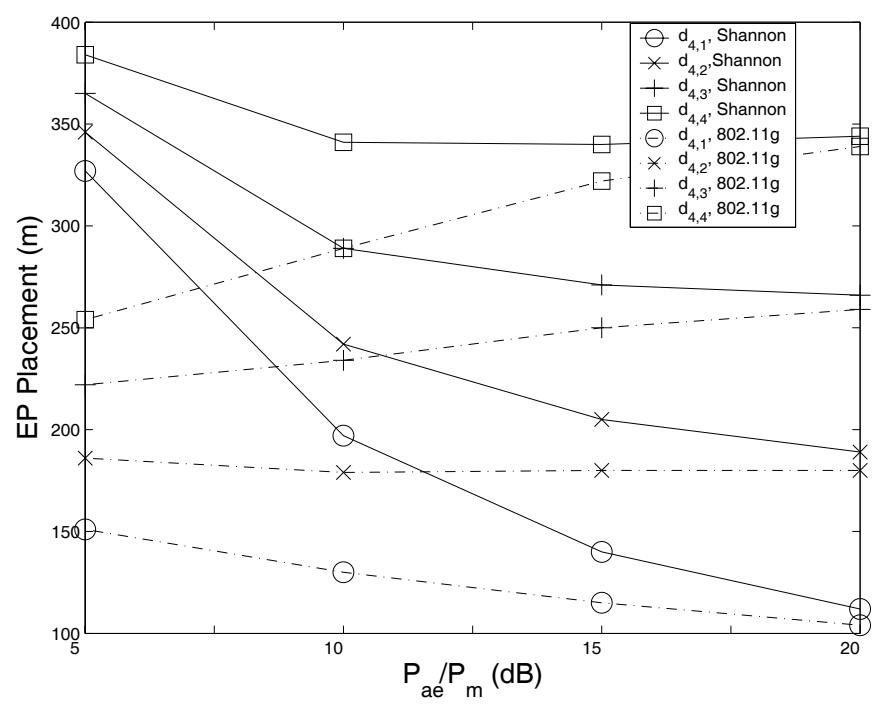

Fig. 16. Optimal placement of 4 EPs with respect to different $P_{a e} / P_{m}$ with Shannon point-to-point channel model and $802.11 \mathrm{~g}$ bit rates with Rayleigh fading channel model.

increases. In this study, the Shannon point-to-point channel model is used to approximate the achievable bit rates of future WLAN. This result suggests that when the mobile hosts are operating in a low power regime, the optimal placements of the EPs will only change slightly when the network is upgraded to a newer standard which can achieve bit rates closer to the Shannon capacity. Since a mobile's battery life is short, it is desired for the mobile hosts to minimize the energy used by its transmitter. Thus, it is reasonable to believe that mobile hosts will be operated in a low power regime in the near future. In such scenario, when a new WLAN standard arises, a network administrator does not have to reinstall the EPs to another locations and the EPs can still provide close to optimal benefit to the network. Moreover, if the EPs are builded by software defined radio, a remote software upgrade is sufficient 


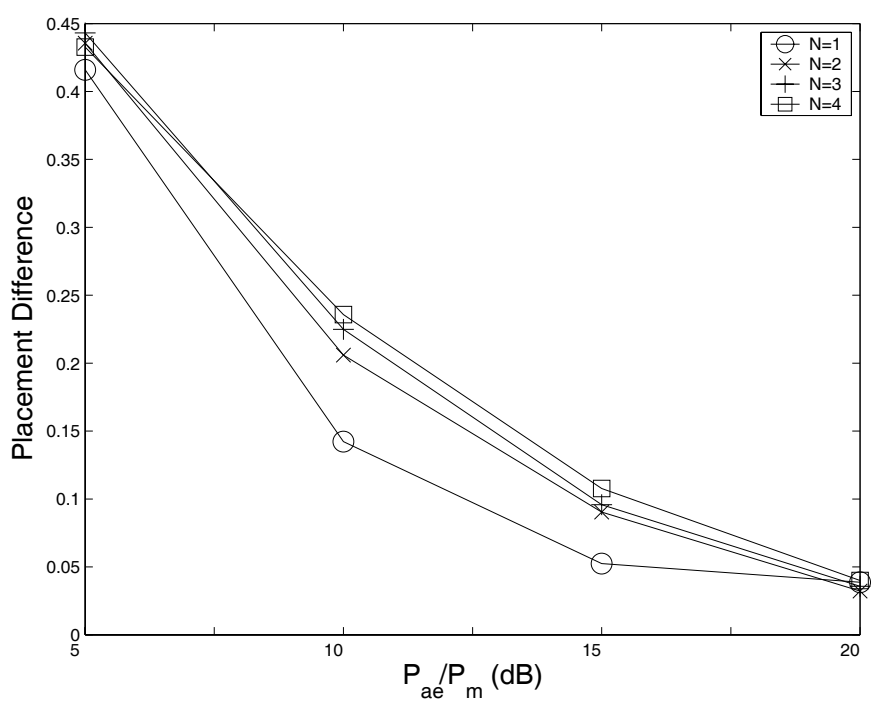

Fig. 17. Average placement difference between the two channel models with respect to $\frac{P_{a e}}{P_{m}}$ ratio under Shannon point-to-point channel model and $802.11 \mathrm{~g}$ bit rates with Rayleigh fading channel model.

to upgrade the EPs to the newest standard. Thus, the laborintensive hardware upgrade routine can be avoided.

\section{Summary of Trends and Observations}

In the range of parameters considered, we observe that the throughput capacity performance gain of utilizing EPs can be significant. Depend on the system parameters and channel model used, the capacity gain of the network range from only a few percent to a few hundred percent. The optimal locations of the EPs are more spread out when the roll off factor of the wireless environment, and the $P_{a e} / P_{m}$ ratio are high. In contrast, when the proportion of downlink data is high, the optimal locations of the EPs are less spread out. The optimally placed EPs are placed closer to the AP when the roll off factor of the wireless environment and the proportion of downlink data is high, while the same is true when the $P_{a e} / P_{m}$ ratio is low.

The performance gain increases when (1) the proportion of uplink data, (2) the $P_{a e} / P_{m}$ ratio, (3) the roll off factor, and (4) the number of EPs increases. As these parameters increase, the beneficial effect of the EP as a wireless power signal booster becomes more significant, which in turn results in better performance gain. In all cases, the effect of diminishing return can be observed when the number of EP increases. Finally, under the current high power regime, the optimal placements obtained by using the two channel models are quite high. In contrast, if the mobile hosts use a low power transmitter, the optimal placement obtained by the two channel model is very small. This suggests that in future WLANs which mobile hosts operate in low power, when the networks is upgrade to a newer standard, the optimal positions of the EPs will only change slightly; thus, reinstallation of the EPs to different locations can be avoided.

\section{CONClusions}

In this work, we have investigated the optimal placement of wireless EPs to maximize the capacity of a heterogeneous WLAN. An efficient optimal EP placement algorithm is proposed. Different channel models can be used in the proposed algorithm. Both the ideal Shannon point-to-point capacity bound and the IEEE $802.11 \mathrm{~g}$ physical layer model with Rayleigh fading are considered. Furthermore, when a practical network with other physical characteristics is deployed, the channel condition can be measured and recorded via site survey, and the proposed algorithm can use the obtained data to derive the optimal EP locations.

Through numerical analysis, we have showed that in most cases, by using optimally placed EPs, the network capacity can be significantly improved. Moreover, for different channel models and network environment, the effects of the AP/EP power to $\mathrm{MH}$ power ratio, network roll off factor, proportion of downlink data, and number of available EPs, with respect to the optimal EP placement and performance gain are quantified. Given a set of network parameters, the proposed algorithm can be used by network designers to compute the optimal placement of EPs and justify the tradeoff between additional hardware cost and system performance gain.

\section{REFERENCES}

[1] D. Gesbert, M. Shafi, D. Shiu, P.J. Smith, and A. Naguib, "From theory to practice: an overview of MIMO space-time coded wireless systems," IEEE Journal on Selected Areas in Communications, vol. 21, no. 3, pp. 281 - 302, April 2003.

[2] E.S. Sousa, "Highly sectorized system for internet wireless access," in in Proc. of IEEE Wireless Communications and Networking Conference (WCNC), Sept. 2000, vol. 1, pp. $153-158$.

[3] H. Wu, C. Qiao, S. De, and O. Tonguz, "Integrated cellular and ad hoc relaying systems: iCAR," IEEE Journal on Selected Areas in Communications, vol. 19, no. 10, pp. 2105-2215, October 2001.

[4] A. Fujiwara, S. Takeda, H. Yoshino, and T. Otsu, "Capacity improvement in a CDMA system using relaying," in in Proc. of IEEE Vehicular Technology Conference (VTC), Sept. 2002, vol. 4, pp. $2371-2374$.

[5] N. Badruddin and R. Negi, "Capacity improvement in a CDMA system using relaying," in in Proc. of IEEE Wireless Communications and Networking Conference (WCNC), Mar. 2004, vol. 1, pp. 243 - 248.

[6] Z. Haas, J. Deng, B. Liang, P. Papadimitratos, and S. Sajama, Wiley Encyclopedia of Telecommunications, John Wiley \& Sons, 2002.

[7] Y. Lin and Y. Hsu, "Multihop cellular: A new architecture for wireless communications," in Proc. of IEEE INFOCOM, Tel-Aviv, Israel, March 2000, vol. 3, pp. $1273-1282$.

[8] S. Mengesha and H. Karl, "Relay routing and scheduling for capacity improvement in cellular WLANs," in Proc. Modeling and Optimization in Mobile, Ad Hoc and Wireless Networks (WiOpt'03), Sophia-Antipolis, France, March 2003.

[9] J. Zhang, B. Li, Q. Zhang, and W. Zhu, "Energy efficient routing in mobile ad-hoc networks: Mobility assisted case," IEEE Transactions on Vehicular Technology, vol. 50, no. 1, Jan. 2006.

[10] R.L. Cruz and A.V. Santhanam, "Optimal routing, link scheduling and power control in multihop wireless networks," in Proc. of IEEE INFOCOM, San Francisco, California, USA, April 2003, vol. 1, pp. $702-711$.

[11] K. Yuen, B. Liang, and B. Li, "A distributed framework for correlated data gathering in sensor networks," to appear in the IEEE Transactions on Vehicular Technology.

[12] S. Mengesha, H. Karl, and A.Wolisz, "Capacity increase of multihop cellular WLANs exploiting data rate adapatation and frequency recycling," Tech. Rep., Technical University Berlin Telecommunication Networks Group, 2003.

[13] J. Zhang, H. Wu., B. Li, and Q. Zhang, "Joint routing and scheduling in multi-radio multi-channel multi-hop wireless networks," in Proc. of IEEE BroadNet, Boston, MA, U.S.A., Oct. 2005. 
[14] N. Esseling, "Extending the range of HiperLAN/2 cells in infrastructure mode mode using forward mobile terminals," in European Personal Mobile Communication Conference 2001, Vienna, Austria, February 2001.

[15] F. Fitzek, D. Angelini, G. Mazzini, and M. Zorzi, "Design and performance of an enhanced ieee 802.11 MAC protocol for multihop coverage extension," IEEE Wireless Communications, vol. 10, no. 6, pp. 30-39, December 2003.

[16] N. Esseling, E. Weiss, and W. Zirwas A. Kramlling and, "A multi hop concept for HiperLAN/2: Capacity and interference," in European Wireless 2002, Florence, Italy, February 2002, pp. 188-189.

[17] X. He, D. Funato, and T. Kawahara, "Delay-sensitive application support in 802.11 based multi-hop network," in Proc. of IEEE Global Telecommunications Conference (GLOBECOM'03), Dec. 2003, vol. 6, pp. $3542-3546$.

[18] E. van der Meulen, "Three-terminal communication channels," Advanced Applied Probability, vol. 3, pp. 120-154, 1971.

[19] A.Gammal T. Cover, "Capacity theorems for the relay channel," IEEE Transactions on Information Theory, vol. 25, no. 5, pp. $572-584$, September 1979.

[20] B. Aazhang M. Khojastepour, A. Sabharwal, "On capacity of gaussian 'cheap' relay channel," in Proc. of IEEE Global Telecommunications Conference GLOECOM'03, San Francisco, California, USA, December 2003, vol. 3, pp. $1776-1780$.

[21] J. Zhang A. Host-Madsen, "Capacity bounds and power allocation in wireless relay channel," IEEE Transactions on Information Theory, June 2005.

[22] A. So and B. Liang, "Effect of relaying on capacity improvement in wireless local area networks," in Proc. of IEEE WCNC, New Orlean, LA, U.S.A., Mar. 2005, vol. 3, pp. 1539 - 1544.

[23] "IEEE standard for information technology, local and metropoliton networks - specific requirements, part 11: Wireless LAN medium access control (MAC) and physical layer (PHY) specifications," Tech. Rep., IEEE Computer Society, 1999

[24] H. Zhu and G. Cao, "On improving the performance of IEEE 802.11 with relay-enabled PCF," ACM/Kluwer Mobile Networking and Applications (MONET), vol. 9, pp. 423-434, 2004.

[25] H. Zhu and G. Cao, "rDCF: A relay-enabled medium access control protocol for wireless ad hoc networks," in Proc. of IEEE INFOCOM, Miami, FL, U.S.A., March 2005, vol. 1, pp. 12-22.

[26] G. Holland, N. Vaidya, and P. Bahl, "A rate-adaptive MAC protocol for multi-hop wireless networks," in Proc. of ACM MOBICOM, Rome, Italy, July 2001, pp. 236 - 251.

[27] B. Sadeghi, V. Kanodia, A. Sabharwal, and E. Knightly, "Opportunistic media access for multirate ad hoc networks," in Proc. of ACM MOBICOM, Atlanta, GA, U.S.A., Sept. 2002, pp. 24-35.

[28] A. Papoulis and U. Pillai, Probability, Random Variables and Stochastic Processes, McGraw Hill, 4 edition, 2002.

[29] T. S. Rappaport, Wireless Communications: Principles and Practice, Prentice Hall, 2 edition, 2001.

[30] T. Cover J. Thomas, Elements of Information Theory, Wiley Series in Telecommunications, 2 edition, 1991.

[31] A. So and B. Liang, "A Lagrangian approach for the optimal placement of wireless relay nodes in wireless local area networks," in Proc. of the International IFIP-TC6 Networking Conference, Coimbra, Portugal, May 2006, pp. 160-172.

[32] "CISCO Aironet 1100 series access point: Data sheet," Tech. Rep., CISCO Systems, 2003.

\section{APPENDIX I: RELAXED VERSION OF OPTIMIZATION PROBLEM (21)(22)}

In Section IV-A, we state that if we replace the binary constraints (20) and replaced by their equivalent linear constraints, i.e., $0 \leq Y_{(x, y),(i, j)} \leq 1$, the resulting problem is a nonlinear problem, and when the relaxed problem is solved to its optimality, $\mathbf{Y}$ must be binary. In fact, we can show that for all solutions with fractional $\mathbf{Y}$, there exist a binary $\mathbf{Y}_{\mathbf{b}}$ which can result in a better (lower) objective value. To show this, let us consider the following.

Let $x_{1}<x_{2}<x_{3}<\ldots<x_{N}$ be constants, and let $\Delta_{i, j}=$ $x_{j}-x_{i}$. Moreover, let $Y_{i}=\{0,1\}$ for $i=1, \ldots, N$ be the decision variables. Let us consider the following optimization problem.

$$
\begin{aligned}
\min _{\mathbf{Y}}: & x_{1} Y_{1}+x_{2} Y_{2}+\ldots+x_{N} Y_{N} \\
& =x_{1} Y_{1}+\left(x_{1}+\Delta_{1,2}\right) Y_{2}+\ldots+\left(x_{1}+\Delta_{1, N}\right) Y_{N} \\
& x_{1}+\Delta_{1,2} Y_{2}+\ldots+\Delta_{1, N} Y_{N} \\
\text { s.t. } & Y_{1}+Y_{2}+\ldots+Y_{N}=1
\end{aligned}
$$

Since $\Delta_{1, j}>0$ for $j=2, \ldots, N$. If we relax the binary constraints to $0 \leq Y_{i} \leq 1$ for $i=1, \ldots, N$, the optimal solution of the above problem is $Y_{1}=1, Y_{2}=0, \ldots, Y_{N}=0$.

Now, let us go back to our original problem:

$$
\begin{array}{r}
\min _{\mathbf{Y}, \underline{d}}: \quad \sum_{x=-\frac{L}{2}}^{\frac{L}{2}} \sum_{y=-\frac{L}{2}}^{\frac{L}{2}} h_{x, y}\left[T_{\text {noep }}(x \Delta, y \Delta) Y_{(x, y),(0,0)}+\right. \\
\left.\sum_{i \in \Lambda} \sum_{j=1}^{N_{i}} T_{e p}^{i}\left(x \Delta, y \Delta, d_{N_{i}, j}^{i}\right) Y_{(x, y),(i, j)}\right] \\
\text { s.t. } \quad Y_{(x, y),(0,0)}+\sum_{i \in \Lambda} \sum_{j=1}^{N_{i}} Y_{(x, y),(i, j)}=1 \quad \forall(x, y)
\end{array}
$$

If we obtain a solution $(\mathbf{Y}, \underline{d})$ and $\mathbf{Y}$ is fractional, we can always obtain a better solution by setting $Y_{(x, y),(i, j)}=1$ to the smallest corresponding $T_{e p}^{i}\left(x \Delta, y \Delta, d_{N_{i}, j}^{i}\right)$ or $T_{\text {noep }}(x \Delta, y \Delta)$ value.

\section{APPENDIX II: CONVEXITY OF TRANSMISSION TIMES}

\section{A. Shannon Point-to-Point Model}

Clearly, for all positive $P, l$, and $\alpha \geq 2, \ln \left[1+\frac{P}{l^{\alpha}}\right]$ is a decreasing function of $l$, and, thus, $T_{s}(l, P, z)$ is an increasing function of $l$. To study the convexity of $T_{s}(l, P, z)$, we need to find its second derivative. Since

$$
T_{s}(l, P, z)=\frac{z}{W \log _{2}\left(1+\frac{P}{N l^{\alpha}}\right)},
$$

we have

$$
\begin{aligned}
& \frac{d^{2}}{d^{2} l} T_{s}(l, P, z) \\
& =\left[\frac{P \alpha z \ln 2}{N W}\right] \frac{\left[2 S \alpha-\ln \left(1+\frac{P}{N l^{\alpha}}\right)\left[(\alpha+1) l+\frac{P}{N}\right]\right]}{\left(l^{\alpha+1}+\frac{P l}{N}\right)^{2}\left[\ln \left(1+\frac{P}{N l^{\alpha}}\right)\right]^{3}} .
\end{aligned}
$$

From (35), the second derivative is not always positive. Thus, some segments of $T_{s}(l, P, z)$ may not be convex. However, for all the parameters that we used, it is clear that the region of interest are almost always convex. Consider Fig. 18.

For all $\alpha, T_{s}(l, P, z)$ has small concavity when $l$ is small. In general, this region is very short especially when $\alpha$ is large or when the transmitter power is small. Therefore, it has a negligible effect on the proposed algorithm especially in scenarios where the employment of EPs is justified. In other cases, where $\alpha$ is small and the transmitter power is large, the concave region does not affect the practical value of the proposed algorithm. First, if the channel attenuation is small or the transmitter power is high, the EPs contribute to only 


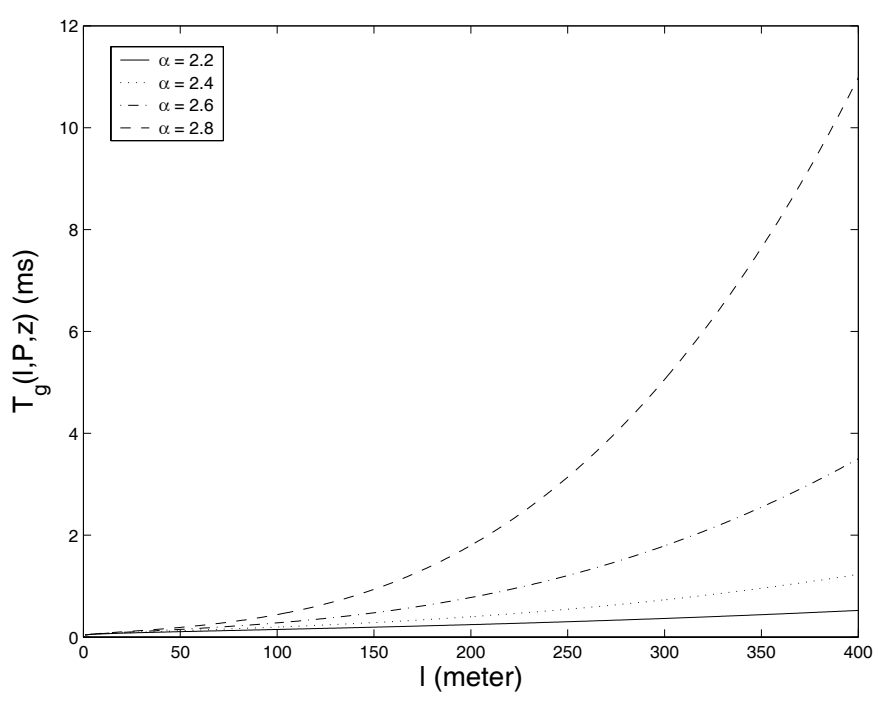

Fig. 18. Packet transmission time with respect to different $\alpha$ with Shannon Point-to-Point channel model for $z=2 k$ bytes, $N_{t}=-90 \mathrm{dBm}, P_{t}=$ $10 \mathrm{dBm}, G_{r}=G_{t}=2.2 d B i, \lambda=0.125 \mathrm{~m}, \mathrm{~W}=20 \mathrm{MHz}$.

marginal or no gain. Hence, using EPs in such scenarios is not justified because of their hardware and maintenance cost. Second, the proposed algorithm is still valid as long as we restrict the solution space such that the condition in Theorem 1 is satisfied. I.e., each EP serve a continuous segment of the network. For general system parameters that we have considered, this condition is always satisfied. Thus, when the ideal model is used and the system parameters reflect the characteristics of a practical urban network, the proposed algorithm can always produce optimal results.

\section{B. IEEE 802.11g with Rayleigh Fading}

By using the CISCO Aironet 802.11g 1100 Series Access Point specifications [32] and the analytical model that we developed, $T_{g}(l, P, z)$ can be obtained. Consider Fig. 19.

Fig. 19 shows the typical curvatures of $T_{g}(l, P, z)$ under different channel environments. As shown in the figure, when $\alpha$ is small to moderate, $T_{g}(l, P, z)$ is convex within the network coverage area. When $\alpha$ is large, $T_{g}(l, P, z)$ becomes slightly concave for large $l$. However, for a large roll off factor, the optimal AP-EP and EP-MH distances are small. Under all practical system parameters that we have considered, the AP-EP and EP-MH distances are never large enough to fall within the range where $T_{g}(l, P, z)$ is concave. Intuitively, when the signal attenuation is high, a network designer should try to minimize the signal travel distance. Thus, any transmitter receiver pair should not be placed too far apart.

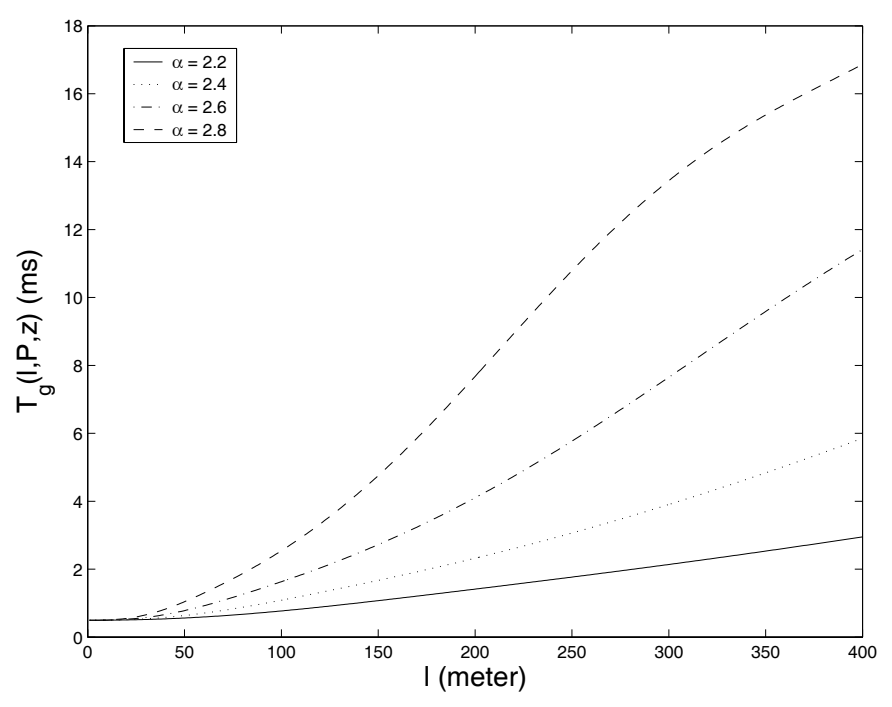

Fig. 19. Packet transmission time with respect to different $\alpha$ with $802.11 \mathrm{~g}$ bit rates with Rayleigh fading channel model for $z=2 k$ bytes, $N_{t}=$ $-90 \mathrm{dBm}, P_{t}=10 \mathrm{dBm}, G_{r}=G_{t}=2.2 \mathrm{dBi}, \lambda=0.125 \mathrm{~m}, \mathrm{~W}=$ $20 M H z$.

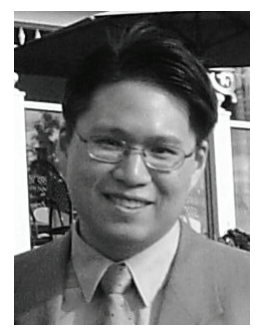

Aaron So (S'04) received B.Sc. (first-class honors) and M.Sc. degrees in Mathematics and Engineering from Queen's University in Kingston, Ontario, Canada in 2002 and 2003 respectively. He joined the $\mathrm{Ph} . \mathrm{D}$. program in the Department of Electrical and Computer Engineering at the University of Toronto in 2003. He received the Ontario Graduate Scholarship in 2003 and 2006, the Ontario Graduate Scholarship in Science and Technology in 2004 and 2005, and the Bell University Labs Scholarship in 2006 toward the completion of his Ph.D. dissertation. His Ph.D. research topic is strategic configurations of relay infrastructure in wireless networks.

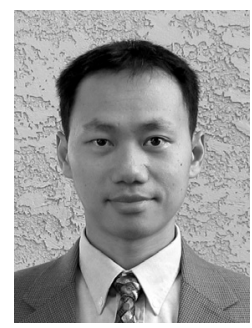

Ben Liang (S'94-M'01-SM'06) received honors simultaneous B.Sc. (valedictorian) and M.Sc. degrees in electrical engineering from Polytechnic University in Brooklyn, New York, in 1997 and the Ph.D. degree in electrical engineering with computer science minor from Cornell University in Ithaca, New York, in 2001. In the 2001 - 2002 academic year, he was a visiting lecturer and post-doctoral research associate at Cornell University. He joined the Department of Electrical and Computer Engineering at the University of Toronto as an Assistant Professor in 2002.

His current research interests are in mobile networking and multimedia systems. He received an Intel Foundation Graduate Fellowship in 2000 toward the completion of his Ph.D. dissertation, the Best Paper Award at the IFIP Networking conference in 2005, and the Runner-up Best Paper Award at the International Conference on Quality of Service in Heterogeneous Wired/Wireless Networks in 2006. He is a senior member of IEEE and a member of ACM and Tau Beta Pi. He serves on the organizational and technical committees of a number of major conferences each year. 